\title{
Impaired emotion perception and categorization in semantic aphasia
}

Nicholas E. Souter ${ }^{\text {a }}$, Kristen A. Lindquist ${ }^{b}$, Elizabeth Jefferies ${ }^{\text {a }}$

${ }^{a}$ Department of Psychology, University of York, York, YO10 5DD, UK

${ }^{\mathrm{b}}$ Department of Psychology and Neuroscience, University of North Carolina, Chapel Hill, NC, 27599-3270, USA

Corresponding Author: Nicholas E. Souter, University of York, Department of Psychology, York, YO10 5DD

Email:nes522@york.ac.uk

Kristen A. Lindquist - kristen.lindquist@unc.edu

Elizabeth Jefferies - beth.jefferies@york.ac.uk

Declarations of interest: none 
Abstract

According to a constructionist model of emotion, conceptual knowledge plays a foundational role in emotion perception; reduced availability of relevant conceptual knowledge should therefore impair emotion perception. Conceptual deficits can follow both degradation of semantic knowledge (e.g., semantic 'storage' deficits in semantic dementia) and deregulation of retrieval (e.g., semantic 'access' deficits in semantic aphasia). While emotion recognition deficits are known to accompany degraded conceptual knowledge, less is known about the impact of semantic access deficits. Here, we examined emotion perception and categorization tasks in patients with semantic aphasia, who have difficulty accessing semantic information in a flexible and controlled fashion following left hemisphere stroke. In Study 1, participants were asked to sort faces according to the emotion they portrayed - with numbers, written labels and picture examples each provided as category anchors across tasks. Semantic aphasia patients made more errors and showed a larger benefit from word anchors that reduced the need to internally constrain categorization than comparison participants. They successfully sorted portrayals that differed in valence (positive vs. negative) but had difficulty categorizing different negative emotions. They were unimpaired on a control task that involved sorting faces by identity. In Study 2, participants matched facial emotion portrayals to written labels following vocal emotion prosody cues, miscues, or no cues. Patients presented with overall poorer performance and benefited from cue trials relative to within-valence miscue trials. This same effect was seen in comparison participants, who also showed deleterious effects of within-valence miscue relative to no cue trials. Overall, we found that patients with deregulated semantic retrieval have deficits in emotional perception but that word anchors and cue conditions can facilitate emotion perception by increasing access to relevant emotion concepts and reducing reliance on semantic control. Semantic control may be of particular importance in emotion perception when it is necessary to interpret ambiguous inputs, or when there is interference between conceptually similar emotional states. These findings extend constructionist accounts of emotion to encompass difficulties in controlled semantic retrieval.

\section{Keywords}

Stroke, Semantic, Aphasia, Emotion, Perception, Cueing 


\section{Introduction}

Semantic cognition underpins our ability to acquire, store and retrieve conceptual information. An outstanding question in this field concerns the nature of the relationship between semantic cognition and emotion. Within the semantic memory literature, the 'hub and spoke' framework proposes that conceptual representations are reliant on interactions between a transmodal hub and modality-specific spokes, resulting in rich heteromodal representations of concepts (Patterson et al., 2007). The anterior temporal lobes (ATL) have been implicated as the site of this hub, while the spokes in this system provide input from sensory modalities including sound and vision, as well as from hedonic valence (Lambon Ralph et al., 2017). Riberto et al. (2019) recently argued that regions connected to the ATL, including the amygdala and orbitofrontal cortex, may act as spokes by generating emotional aspects of heteromodal representations. Under this framework, valence information is therefore an important feature of concepts.

The constructionist model of emotion considers the inverse of this relationship; namely the contribution that language and conceptual knowledge make to the perception of emotion (Lindquist et al., 2015). Perceiving facial muscle portrayals of emotion results in rapid and automatic activation of emotion concepts (Osborne-Crowley et al., 2019; Preston, 2007). Moreover, temporarily disrupting access to emotion labels (e.g., "angry") using semantic satiation impairs the perception of facial emotion portrayals (Gendron et al., 2012; Lindquist et al., 2006), indicating an involvement of language in emotion processing even when not explicitly necessary. Beyond language, Brooks and Freeman (2018) found that ratings of conceptual similarity between pairs of emotion categories (e.g., "fear" and "anger") predicted perceived perceptual similarity of faces corresponding to these categories. Similarly, exposure to emotion labels is thought to aid children's formation of emotion concepts, which expedites their perception of discrete facial emotions (Hoemann et al., 2019). Interoceptive models similarly argue that physiological valenced feelings and conceptual information interact to construct our interpretation of emotion categories (Zhou et al., 2021). These models and studies all suggest that accessing knowledge about emotional states facilitates the perception of discrete emotional portrayals.

This account implies that impairments in semantic cognition should also manifest as impairments in emotion perception. Patients with semantic dementia (SD) experience a progressive loss of heteromodal conceptual knowledge following atrophy focussed on 
bilateral ATL (Bozeat, Lambon Ralph, et al., 2000; Mummery et al., 2000), often accompanied by emotional difficulties including apathy, diminished empathy, and emotional withdrawal (Bozeat, Gregory, et al., 2000; Hodges \& Patterson, 2007). To test predictions of the constructionist model, Lindquist et al. (2014) studied the ability of SD patients with left ATL atrophy to categorize pictures of facial emotion portrayals. Participants were asked to sort photographs of faces portraying six English language categories of emotion (anger, disgust, fear, happiness, sadness, and neutral) into as many piles as meaningful. SD patients spontaneously created three to four piles reflecting the valence of the facial portrayals (i.e., pleasant, unpleasant, and neutral), whereas healthy age-matched controls created on average seven to eight categories reflecting specific emotions. Providing patients with exemplar facial portrayals improved their ability to sort by emotion, whereas the presence of written emotion category labels did not. Notably, SD patients were able to sort the faces into six categories by identity, suggesting that their errors did not reflect general cognitive impairment. Kumfor et al. (2018) similarly demonstrated impaired ability to decode facial or bodily portrayals of emotion in SD, regardless of task demands. The failure of conceptual anchors to boost categorization performance is consistent with SD patients' highly predictable semantic deficits when the same concepts are probed using different tasks (Bozeat, Lambon Ralph, et al., 2000; Jefferies \& Lambon Ralph, 2006) and the weak impact of cues on semantic retrieval (Jefferies et al., 2008). Evidence suggests that emotion processing deficits in SD are partially explained by performance on verbal semantic tests (Miller et al., 2012), supporting the view that these deficits are attributable to semantic impairment. It has also been argued that this impairment arises in part due to strong connectivity of ATL with key emotion processing regions such as the amygdala (Rosen et al., 2002). Together, these studies suggest a loss of conceptual knowledge following semantic storage deficits in SD impairs the ability to perceive specific emotional states.

While SD patients show highly consistent semantic storage deficits across tasks, patients with semantic aphasia (SA) have semantic access deficits that result in deregulated conceptual retrieval across verbal and non-verbal tasks following infarcts in left inferior lateral prefrontal and/or temporoparietal cortices (Corbett et al., 2011; Jefferies, 2013; Jefferies \& Lambon Ralph, 2006). Moreover, whereas SD patients show relatively good performance on tests of non-verbal reasoning, semantic deficits in SA often co-occur with executive dysfunction (Thompson et al., 2018). SA patients' access to specific concepts is dependent on task demands (Jefferies \& Lambon Ralph, 2006): they can show near-normal 
performance when the control demands of semantic tasks are minimised and have more severe deficits when meanings are ambiguous or strong distracters are presented (e.g., Montefinese et al., 2020; Noonan et al., 2010; Thompson et al., 2017). The impairment in SA can also be ameliorated by providing cues which constrain the meaning of target concepts, reducing the need for controlled retrieval. For instance, phonemic cues facilitate picture naming (Jefferies et al., 2008) and sentence contexts support semantic decisions (Noonan et al., 2010). Lanzoni et al. (2019) found facial emotion portrayals also influence semantic access in SA: positive interpretations of ambiguous words are more accessible following smiling faces, while negative interpretations become less accessible. SA patients can therefore be cued to access concepts that they often fail to retrieve, suggesting that, unlike in $\mathrm{SD}$, the concepts themselves are not degraded. This qualitative difference is thought to reflect differences in the distribution of atrophy and infarcts in SD and SA: ATL is a watershed region with a dual blood supply (Phan et al., 2007) and consequently heteromodal conceptual knowledge may be relatively invulnerable to damage from stroke.

In line with this dissociation between SD and SA, the controlled semantic cognition (CSC) framework (Jefferies, 2013; Lambon Ralph et al., 2017; Rogers et al., 2015) proposes that semantic control processes interact with conceptual representations to support the flexible retrieval of appropriate knowledge. Converging neuroimaging and neurostimulation studies of healthy participants implicate the left inferior frontal gyrus (LIFG) and posterior middle temporal gyrus (pMTG), often damaged in SA, in controlled semantic retrieval (Badre et al., 2005; Davey et al., 2016; Hallam et al., 2016; Thompson-Schill et al., 1997). This leftlateralised network is thought to constrain the retrieval of both verbal and nonverbal information (Krieger-Redwood et al., 2015) and can bias the interaction of sensory-motor spokes with the hub in accordance with the information required (Chiou et al., 2018; Jefferies et al., 2019; Zhang et al., 2021). Neuroscientific studies show that the semantic control network is partially distinct from both multiple demand cortex, which supports domaingeneral cognitive control (Davey et al., 2016; Diachek et al., 2020; Gao et al., 2021; Jackson, 2021; Noonan et al., 2013), and default mode network regions that underpin more integrative or automatic aspects of conceptual retrieval (e.g., ATL and AG; Humphreys et al., 2015; Lanzoni et al., 2020; Vatansever et al., 2017).

The relevance of this distinction between conceptual representation and semantic control processes for emotion perception has not been investigated to date, although Robertson et al. (1999) observed impaired emotion categorization in a single aphasia patient 
and cited conceptual difficulties as a possible cause. More generally, evidence from healthy adults has suggested involvement of top-down processes in the perception of discrete emotions, as demonstrated by tasks implementing categorical emotion primes (Carroll \& Young, 2005; Doyle et al., 2021; Zemack-Rugar et al., 2007) and those manipulating the context in which emotional facial portrayals appear (Aviezer et al., 2008). In the current study, we investigated the effects of deregulated semantic retrieval in SA patients on perception of emotion from facial portrayals. If activation of emotion concepts is automatic upon perceiving certain configurations of facial muscle movements in others (OsborneCrowley et al., 2019; Preston, 2007), we would not expect controlled semantic retrieval to be required for this process. Consequently, the perception of emotion may be unimpaired in SA. Alternatively, if perceptual processing of emotion is influenced by the accessibility of conceptual knowledge (Gendron et al., 2012; Lindquist et al., 2006), impaired retrieval may interfere with the activation of relevant emotional states, particularly when in the presence of competing and conceptually similar portrayals. This hypothesis is supported by evidence that the LIFG, a key semantic control region (Jackson, 2021), is implicated in the discrimination of emotional states (Jastorff et al., 2016).

In Study 1, we adopted the procedures used by Lindquist et al. (2014) with SD patients, and collected a comparative sample of SA cases. This paradigm allowed us to assess the effects of impaired semantic control on emotion categorization with minimal constraints, and in the presence of numbers, written words, and exemplar faces, provided as sorting anchors. In the unconstrained free sort, the need to categorise faces according to emotion (e.g., 'angry') as distinct from related distractors (e.g., sad or disgusted faces) is expected to tax semantic control. The need to internally constrain categorisation was expected to be reduced by sorting anchors, and consequently SA patients might benefit more from their availability than healthy comparison participants. Study 2 examined the effects of emotional prosody on the categorisation of facial emotion portrayal in an alternative forced choice design. Prosody was either consistent with the portrayed facial emotion (cue condition) or inconsistent (miscue condition). Within-valence miscues (e.g., sad prosody preceding an angry face) were expected to impair performance, since they required the suppression of information that was irrelevant but conceptually similar to the target concept. SA patients were expected to be impaired relative to comparison participants, especially following miscues. Overall, these studies (i) provide further evidence that semantically-impaired participants are impaired at discriminating portrayals of distinct emotional categories yet are 
not impaired at recognising positive and negative valence and (ii) show that, in both SA patients and comparison participants, emotion recognition is sensitive to the requirement to control conceptual processing.

\section{Method}

\subsection{Participants}

The overall sample consisted of seven patients, and 33 healthy comparison participants. Patients were recruited from communication support groups across Yorkshire. All patients had aphasia following left hemisphere stroke and showed semantic impairment across verbal and non-verbal tasks (see section 2.3.). These inclusion criteria were adopted since heteromodal semantic impairment following left hemisphere stroke has been shown to be associated with deregulated access to conceptual information, as opposed to a degraded store of conceptual knowledge (Jefferies, 2013). Comparison participants were healthy adults broadly similar to the patients for age and level of education, with no history of psychiatric or neurological disorder. As a subset of participants from Study 1 participated in Study 2, respective demographics are reported separately in sections 3.1.1. and 4.1.1.

\subsection{Lesion Analyses}

Three patients (P4, P6, and P10) had structural MRI scans at the York Neuroimaging Centre. These scans underwent brain extraction and registration to MNI space in ANTs (Avants et al., 2011) using a template from the OASIS Brain Project (https://www.oasisbrains.org/; Marcus et al., 2010). Each patient's lesion was manually traced in MRICron. Care was taken to avoid implicating enlarged ventricles or sulci. Manually segmented lesion locations for these three patients are presented in Figure 1. It was not possible to obtain structural scans for the remaining four patients, due to contraindications for scanning and/or closure of scanning facilities during the COVID-19 pandemic. Clinical acute-stage scans were obtained for P16 (MRI) which showed evidence of a left frontal lesion, and for P12 (CT) which showed evidence of a left fronto parietal lesion. These data suggest that at least five out of seven patients had damage to left lateral prefrontal cortex, known to be important for semantic control. 


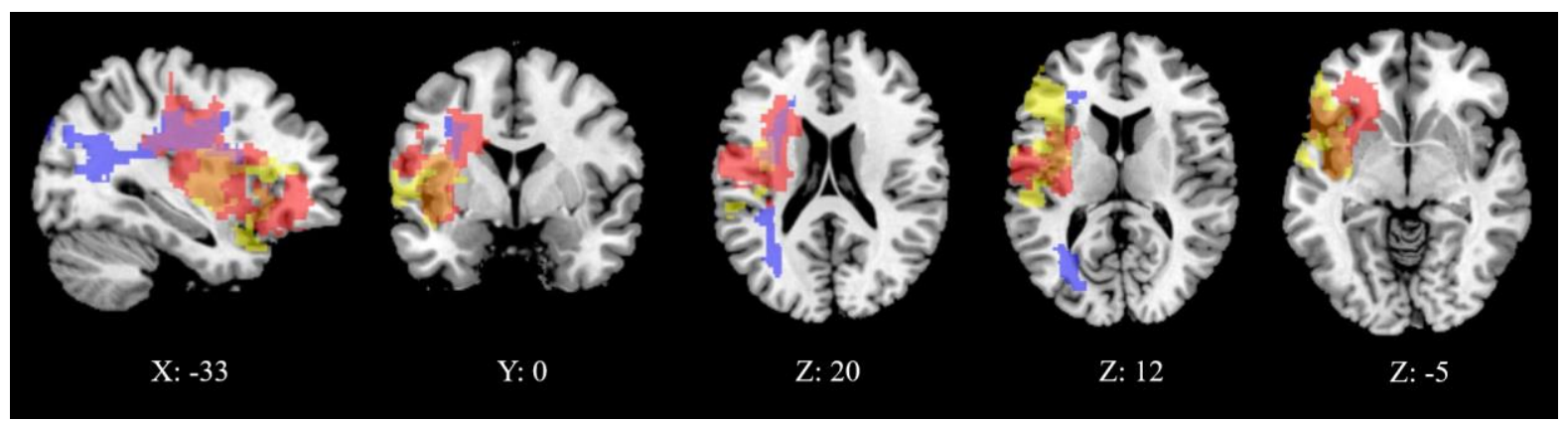

Figure 1: Manually segmented lesion location for P4 in red, P6 in blue, and P10 in yellow. Overlap of P4 and P6 can be seen in purple, P4 and P10 in orange, and overlap of all three in dark pink. There is no exclusive overlap of P6 and P10 here. The lesions for all three patients impact the LIFG. P4's lesion extends to the left pMTG. Voxel coordinates are displayed under each slice.

\section{*COLOUR NEEDED IN PRINT*}

\subsection{Background neuropsychological testing}

Patients completed a series of neuropsychological assessments, testing language, memory, visuospatial function, executive function, and semantic cognition. The protocol used here was similar to that described in Stampacchia et al. (2018). Each patient's performance on the background neuropsychology measures is shown in Supplementary Table 1, while Supplementary Table 2 provides data on tests of semantic cognition. The Supplementary Materials also provide task descriptions and an interpretation of the sample's performance on these measures.

Patients presented with largely preserved word repetition, but impairments in verbal fluency and working memory. Visuospatial processing was largely preserved across the sample. Across a series of executive function tests, three patients showed some evidence of impairment. While the extent of executive dysfunction in SA is debated (see Thompson et al., 2018, and Chapman et al., 2020, for alternative viewpoints), the current sample may be less impaired than previous studies from our group, due to the need for patients to meet the constraints associated with remote testing (e.g., self-directed computer use) during the COVID-19 pandemic.

Patients showed a variable degree of impairment on core tests of semantic cognition. As expected, impairment was highest on tests that had larger semantic control demands, with patients showing effects of cues and miscues, competition from strong thematic distractors, and difficultly retrieving subordinate conceptual information in both the verbal and non- 
verbal domains. Each patient showed impairment on at least one verbal and one non-verbal test of semantic control. This is consistent with the view that patients with SA have multimodal impairment in the manipulation of knowledge for the purpose of symbolic processing, as first outlined by Head (1926). The degree of impairment for each patient was derived from a semantic control composite score, extracted from a larger sample of SA patients (Souter et al., Unpublished results; $\mathrm{N}=17$, including the current sample) using principal components analysis (PCA) with oblique rotation. This component reflects performance on tasks with high semantic control demands, including word and picture versions of the Camel \& Cactus Test (Bozeat, Lambon Ralph, et al., 2000), the ambiguity task (Noonan et al., 2010), the synonym judgement task (Samson et al., 2007), and the object use task (Corbett et al., 2011). For each patient, regression scores for this component were extracted, with lower scores indicating greater impairment. Each patient's composite scores are reported in Supplementary Table 2, and the full results of this PCA are presented in Supplementary Table 3.

\section{Study 1: Emotion portrayal categorization}

\subsection{Method}

\subsubsection{Participants}

The Study 1 sample included seven patients (four female) with a mean age of 57.0 $(\mathrm{SD}=9.2)$, a mean age of leaving education of $18.4(\mathrm{SD}=3.8)$ and a mean of 11.5 years $(\mathrm{SD}$ $=5.4$ ) since their most recent stroke. We also collected data from 33 healthy comparison participants (20 female) with a mean age of $67.7(\mathrm{SD}=9.5)$, and a mean age of leaving education of 20.8 ( $\mathrm{SD}=3.0)$. Patients and comparison participants did not significantly differ on age when leaving education: $\mathrm{t}(38)=-1.8, p=.078$. The comparison participants were significantly older than the patients: $\mathrm{t}(38)=-2.7, p=.010$ (this difference should underestimate impairment in the patients). In the original study on SD (Lindquist et al., 2014), patients were compared to comparison participants in the emotion free sort task only, while we collected control data for all sorting tasks to establish how word and face anchors aid SA patients, relative to comparison participants. Informed consent was obtained for all participants. 


\subsubsection{Design}

This study used a repeated measures design, with all participants completing four sorting tasks with varying levels of constraint; free sort, number anchored sort, word anchored sort, and face anchored sort. These tasks were completed over the course of a single session. No limitations were put on timing, but administration typically lasted between 40 and 60 minutes.

\subsubsection{Stimuli}

As in the original paradigm (Lindquist et al., 2014), stimuli were taken from the IASLab Face Set ${ }^{1}$ (https://www.affective-science.org/face-set.shtml). A full list of stimuli (by identity code) is provided in Supplementary Table 4. We replicated the short version of the sorting tasks used in the original publication, with six identities (half male, half female) all portraying facial muscle configurations associated with the six English language emotion categories of anger, sadness, disgust, fear, happiness, and neutral. This resulted in 36 stimuli overall. One male identity from the original paradigm was substituted for a novel identity, as perceptual similarity between two identities was found to cause confusion in the free sort task (see section 3.1.4.2.). Two patients (P10, P11) and three comparison participants who had completed the full study with the male identity used in the original paradigm, were retested on the identity free sort task with the updated stimuli. These participants were not retested on the remaining conditions, as practice effects (i.e., knowledge of the number and contents of the emotion categories) in the emotion free sort condition would have been unavoidable.

\subsubsection{Procedure}

Due to COVID-19 social distancing measures, the procedure from Lindquist et al. (2014) was adapted for online remote testing. Stimuli were presented online using Google Slides, while the researcher and participant conversed on the video conferencing software Zoom (Zoom Video Communications Inc., 2016). Both the participant's and researcher's video were turned off, to prevent them obscuring the stimuli. By default, the researcher shared their screen to show the stimuli on Google Slides, and gave the participant remote control of their cursor. This allowed participants to click and drag the stimuli around the screen. If technical issues arose, participants were sent a direct link to the Google Slides document, allowing them to move the stimuli around themselves while the researcher viewed the same document, still speaking to the participant on Zoom or by telephone. One patient

\footnotetext{
${ }^{1}$ Development of the Interdisciplinary Affective Science Laboratory (IASLab) Face Set was supported by the National Institutes of Health Director's Pioneer Award (DP1OD003312) to Lisa Feldman Barrett.
} 
(P6) was unable to engage with online testing. In this case, physical stimuli were printed, laminated, and sent to P6, who then completed the study while on a Zoom call with the researcher. Figure 2 provides a summary of the Study 1 stimuli and procedure, including examples of stimuli, the layout used in each task, and the order these tasks were completed in. 


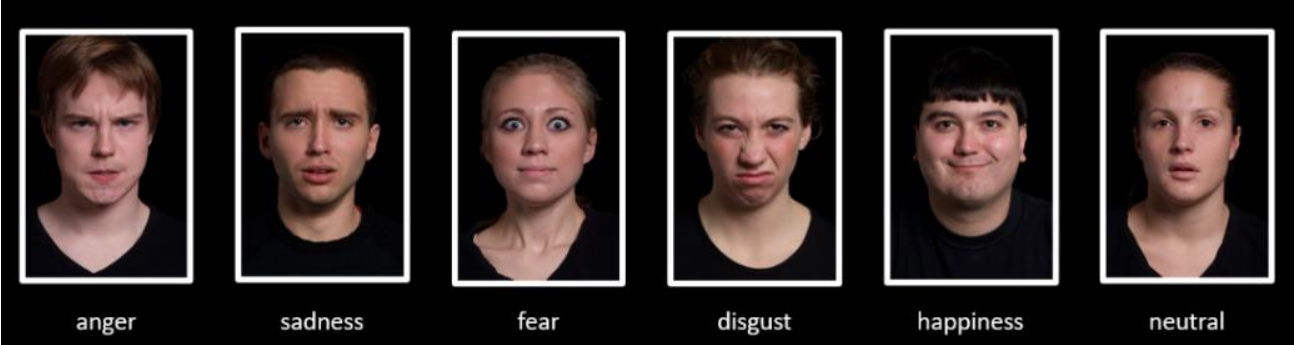

(1)
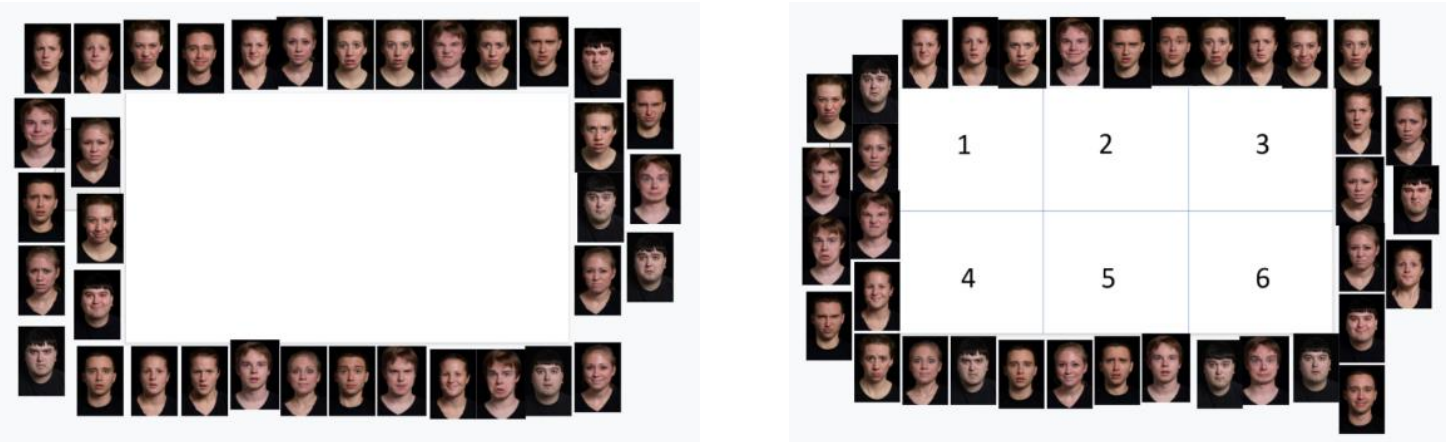

(2)

(3)
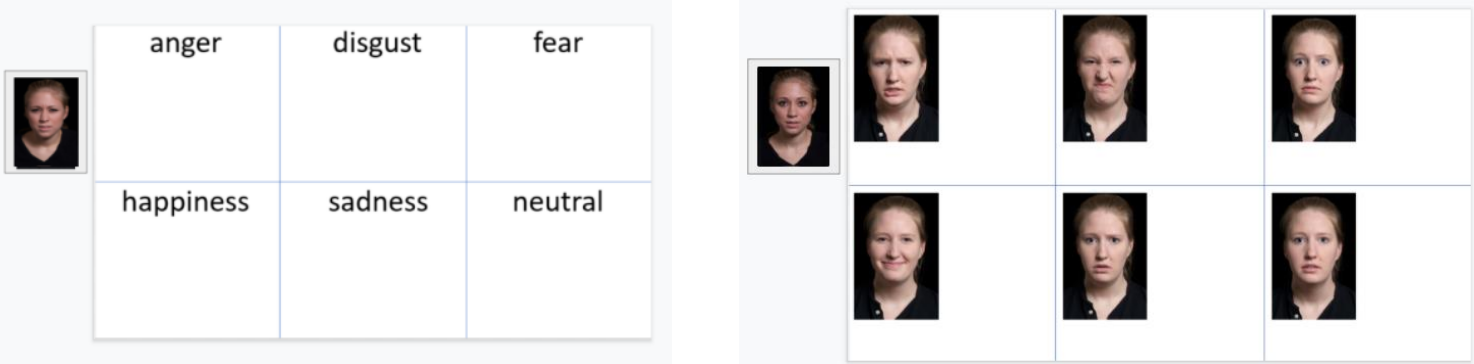

(4)

(5)

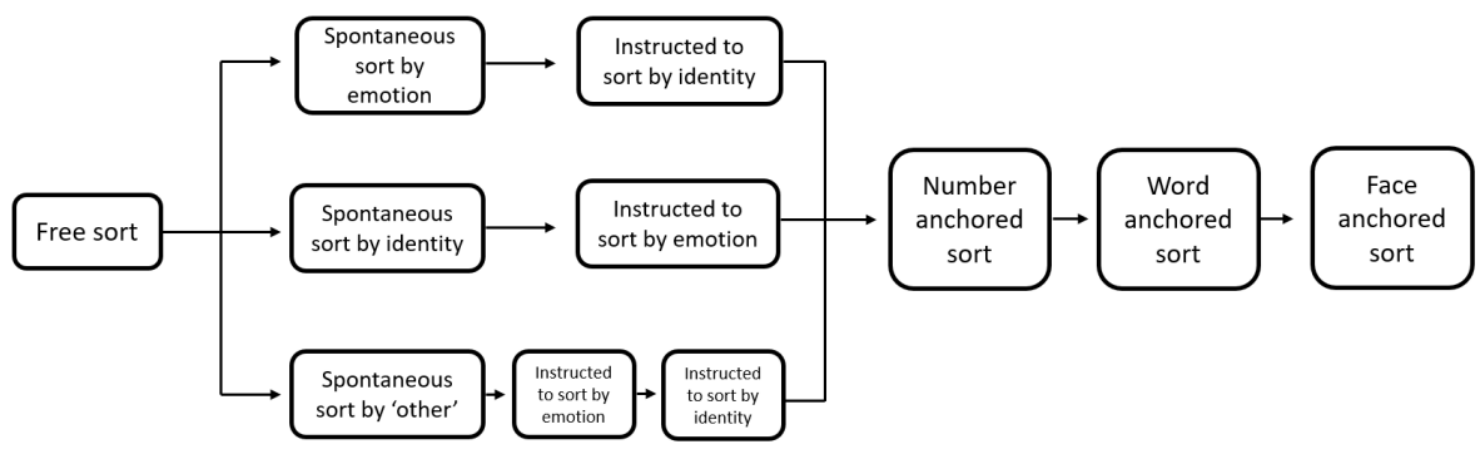

(6)

Figure 2. A summary of the Study 1 stimuli and procedure, including (1) examples of stimuli, layout of stimuli during (2) free sort (including emotion and identity free sort), (3) number anchored sort, (4) word anchored sort, and (5) face anchored sort tasks, and (6) the order these tasks were completed in. 


\subsubsection{Emotion free sort}

The stimuli were distributed randomly around the outside of the screen, with the center of the screen left clear to allow room for creating piles (see Figure 2.2). Participants were told: "On this slide there are a series of pictures spread out. What I want you to do is organize them into groups that are meaningful to you. You can create as many piles as you need to. At the end, each pile should be sorted so that one type of picture only is included in there. It is sometimes helpful to look through the set of pictures first before you begin sorting. This is not timed, so feel free to take as long as you need. You can also change the piles while you are sorting or at the end-it is up to you. Do you have any questions?". Participants were told that the researcher was unable to give further instructions, but that there were no wrong approaches. After sorting had finished, participants were asked "Can you tell me about how you sorted the pictures?". Of the patients, four sorted spontaneously by emotion, two by identity, and one by gender. Of the comparison participants, 22 sorted spontaneously by emotion, nine by identity, one by gender, and one by attempting to organise faces symmetrically across the screen. Participants who had not initially sorted by emotion were then told; "The people on this slide are all feeling something. Some of them feel the same way and some of the people feel differently. What I want you to do now is to sort the faces in the pile based on how the people feel. You should create piles where each person feels exactly the same way. At the end, each pile you've made should have pictures of only people who feel the same way. Are these instructions clear?". Participants who spontaneously sorted by gender or using other irrelevant factors were explicitly instructed to sort by emotion (as above) and then to sort by identity (as below). Once participants had sorted by emotion they were asked, for each pile; "What are the people in this pile feeling?". Participants were prompted to provide a specific emotion adjective to each pile. At the end of each sorting task, participants were advised to check over their piles and move any stimuli they believed they had sorted incorrectly. There was no difference in overall emotion free sort accuracy between participants who first sorted by emotion, and those who first sorted by identity/other categories [patients: $\mathrm{U}=2.0, p=.154$, comparison participants: $\mathrm{U}=103.0, p=.491$ ], suggesting that performance was not affected by increased exposure or explicit instructions.

\subsubsection{Identity free sort}

As noted above, some participants spontaneously sorted the faces according to identity. In cases where they had not, following the emotion free sort task, they were shown the screen described above (Figure 2.2) and were told; "On this slide there are pictures of a 
bunch of people. There are several pictures of each person in the pile. What I would like you to do is to sort the people in this pile into new piles based on their identity. You can create as many new piles as you need to. At the end, each pile you've made should have pictures of only one person in it. Please go as slowly as you need to. This will not be timed. Feel free to examine the images before you begin sorting. Do you have any questions before you begin?". This was intended to act as a control task, to identify cognitive or visual difficulties that could affect emotion categorization.

\subsubsection{Number anchored sort}

Following the free sort task, participants were explicitly told to sort stimuli by emotion into six distinct piles. The layout was the same as used for the free sort tasks, with the exception that boxes numbered one to six were included in the middle of the screen to serve as anchors (see Figure 2.3). Participants were instructed; "Now I want you to again sort these pictures based on feeling, but this time I am going to ask you to make 6 piles. I have 6 different numbers that I have laid out for you so that you can keep track of how many piles you create. Again, I want you to sort based on feeling. In each pile, there should be only people who feel the same way. At the end, each pile you've made should have pictures of only people who feel the same way. Please go as slowly as you need to. This will not be timed. Feel free to examine the images before you begin sorting. Do you have any questions before you begin?". All participants followed the instructions and made six piles, except for one patient (P10) who indicated they were unable to perceive a sixth emotional state and finished with five piles.

\subsubsection{Word anchored sort}

Participants were then again explicitly asked to sort the faces by emotion, this time with the aid of word anchors which labelled what the faces in each pile should feel. In this case, stimuli were not spread around the screen but appeared one at a time in an area on the left of the screen, in a randomised order (see Figure 2.4). This was done as participants were now sorting the stimuli to specific anchors rather than to each other, which was anticipated to be easier to perform when stimuli appeared one at a time. Each stimulus remained on screen after it had been initially sorted and, as with prior conditions, participants were free to change their categorization of a given stimulus at any point during the condition. Participants were instructed; "Now I want you again sort these pictures based on feeling, but this time I am going tell you what should be in each of the 6 piles. There are 6 words laid out here so that you can keep track of the piles. Again, I want you to sort based on feeling. In each pile, there 
should be only people who feel the same way. I want you to sort into this pile, people who feel angry. In this pile, I want you to sort people who feel disgusted. In this pile, I want you to sort people who feel fearful. In this pile, I want you to sort people who feel happy. In this pile, I want you to sort people who feel sad. In this pile, I want you to sort people who feel neutral. At the end of your sorting, each pile you've made should have pictures of only people who feel the same way. Please go as slowly as you need to. This will not be timed. Feel free to examine the images before you begin sorting. Do you have any questions before you begin?".

\subsubsection{Face anchored sort}

In the final task, participants were again explicitly asked to sort stimuli by emotion, this time matching them to exemplar facial portrayals of each of the six English language emotion categories. Example pictures were of a female actor taken from the IASLab Face Set who was posing facial muscle movements prototypically associated with the six emotion categories. These six anchors were presented on the slide's background and were made slightly larger than the stimuli to be sorted, to help participants keep track of the reference picture. Again, stimuli appearing one at a time on the left of the screen in a randomised order (see Figure 2.5). Participants were instructed; "Now I want you to again sort these pictures based on feeling, but this time I am going to start the piles for you. Here are six different pictures of the same woman. The woman feels differently in each of the pictures. Again, I want you to sort the pile of pictures into these already started piles based on feeling. In each pile, there should be only people who feel the same way. At the end of your sorting, each pile you've made should have pictures of only people who feel the same way. Please go as slowly as you need to. This will not be timed. Feel free to examine the images before you begin sorting. Do you have any questions before you begin?".

\subsubsection{Data Analysis}

For each participant, the number of piles created in the emotion free sort task was recorded, reflecting categorization of emotional portrayals with minimal explicit instruction. We also computed accuracy for each participant in each sorting task. For the emotion free sort number anchored sorting tasks, errors were classified as cases where a face was sorted into a pile where the dominant portrayal was of a different emotion, or into piles with no one dominant portrayal. A pile was classified as having a dominant portrayal when at least $50 \%$ of its faces corresponded to one emotion category. In the word anchored sort and face anchored sort tasks, errors were defined as cases where a stimulus was not placed in the pile of its conceptual or perceptual equivalent, regardless of the dominant portrayal of faces 
placed in that pile. Error types were classified in terms of whether they were within-valence (e.g., both involving negatively-valenced states, such as mistaking a sad portrayal as angry) or across-valence (e.g., mistaking a neutral portrayal as sad, or a happy portrayal as fearful). Full error classification data is provided in Supplementary Table 5. Accuracy in the identity sort control task was also assessed. Errors were classified as cases where a face was sorted into a pile of pictures with a different dominant identity, or a pile with no dominant identity. A pile was classified as having a dominant portrayal when at least $50 \%$ of its faces corresponded to one identity. While in the original paradigm (Lindquist et al., 2014) accuracy was graphically presented in terms of ' $\%$ total errors', for the sake of consistency with Study 2 we present ' $\%$ correct' for each task.

Each patient was classified as being either impaired or not impaired in terms of their overall accuracy in each emotion sorting task. This was done using Singlims (Crawford et al., 2010), which compares individual patient scores to the respective mean and standard deviation of a comparison sample to estimate degree of impairment. Singlims was also used for each individual error type for each patient in each task (e.g., to reflect whether an individual patient made an abnormally high number of negative within-valence errors in each task; reflected in Supplementary Table 5). In each case, one-tailed $p$-values were interpreted against a significance threshold of .05 .

Finally, to compare performance in the free sort task with each type of anchor across the two groups, mixed effects logistic regressions were used. Three models were created in $\mathrm{R}$ (R Core Team, 2020), comparing the initial emotion free sort task with performance following each anchor type (numbers, words, and faces). We used separate models for each task comparison rather than an omnibus model due to the fact that this study was comprised of four distinct tasks, tested separately with varying instructions (in contrast, Study 2 below presented multiple conditions within one experiment). For each model, group and task were entered as categorical fixed factors, and participant identity as a random factor. Introducing this random intercept allows for these models to systematically account for variability attributable to participant identity, and therefore to better isolate variability caused by the fixed factors of interest (Bell et al., 2019). We modelled each individual stimulus in each task, coded as having been sorted either correctly or incorrectly. All models were fit by maximum likelihood, based on Gaussian Hermite approximation. Models were run using the lme4 package (version 1.1-25; Bates et al., 2015). As these are logistic models, estimate coefficients reflect log transformation of odds ratios (Larsen et al., 2000). Likelihood ratio 
tests were used to look for significant main effects or interactions of task by group for each model. These tests compared two nested models using the chi-square distribution, to determine whether removing a predictor (e.g., group) significantly changed the overall model. Using this same procedure, we modelled accuracy on the identity sort control task, including group and emotion category as fixed effects, and participant identity as a random factor. All data for Study 1 are publicly available on Mendeley Data (https://data.mendeley.com/datasets/zwxxscny6x/3).

\subsection{Results}

\subsubsection{Sorting based on identity}

Three of the seven patients (P6, P12, P15), and five of the 33 comparison participants made some mistakes on the identity sort control task, even though all participants in the original paradigm (Lindquist et all., 2014) performed this task perfectly (under different viewing conditions). However, there was no difference in accuracy in emotion free sort for participants who had and had not made errors in the identity free sort task [patients: $U=2.5$, $\mathrm{p}=.212$, comparison participants: $\mathrm{U}=34.5, \mathrm{p}=.074]$. Furthermore, mixed effects logistic regression found no effect of group on identity sorting accuracy (see Supplementary Table 7), showing that the SA group did not present with domain-general deficits in sorting in a control task that did not involve accessing categorical representations of emotion.

\subsubsection{Sorting based on emotion portrayal}

On average, comparison participants created $5.7(\mathrm{SD}=1.4)$ piles in the emotion free sort task, with 58\% producing six or seven piles. This is similar to Lindquist et al. (2014), where $61 \%$ of older adults produced six or seven piles. Of the SA patients, three created four piles, two created five, one created six, and one created seven. In Lindquist et al. (2014), the three SD patients all produced either three or four piles reflecting hedonic valence of the portrayals (pleasant, unpleasant, neutral). Therefore, while some SA patients appeared similar to SD patients in terms of perceiving limited discrete emotion states, others identified more categories (although this did not always correspond with greater accuracy, see Supplementary Table 5).

Next, we considered the emotion labels provided by SA patients for all piles in the emotion free sort task. Of the piles which were represented by one dominant emotion, 70\% were attributed the correct emotion label or a largely appropriate alternative to it (e.g., a 
happy pile labelled as 'pleasure', or a neutral pile labelled as 'normal'). The majority of correct labels were for piles of happy or neutral faces, when within-valence discrimination was not necessary. Within-valence labelling errors (e.g., an angry pile labelled as 'fear') occurred in $25 \%$ of cases, and there was only one case of across valence error (a fearful pile labelled as 'happy' by P16). SD patients in the original sample from Lindquist et al. (2014) were similarly accurate in labelling piles as happy or neutral but were sometimes vague in their labelling of negative piles, using labels such as "rough" or "not up". Such vague descriptions were rare in the current sample.

Each patient's overall accuracy (percent correct) for each sorting task, as well as group averages, can be seen in Figure 3. Due to the small patient sample size, it was not possible to calculate correlations between task performance and degree of semantic impairment. Instead, patients in Figure 3 are ordered from most to least impaired (from left to right), allowing for the visualisation of effects of semantic impairment on performance. As determined by Singlims analysis, six of the seven patients were impaired in at least one task, as determined by a significantly lower accuracy when compared to comparison participants. The only patient not to show evidence of emotion discrimination impairment was the one with the mildest semantic impairment (on the basis of the semantic control composite score, see section 2.3).

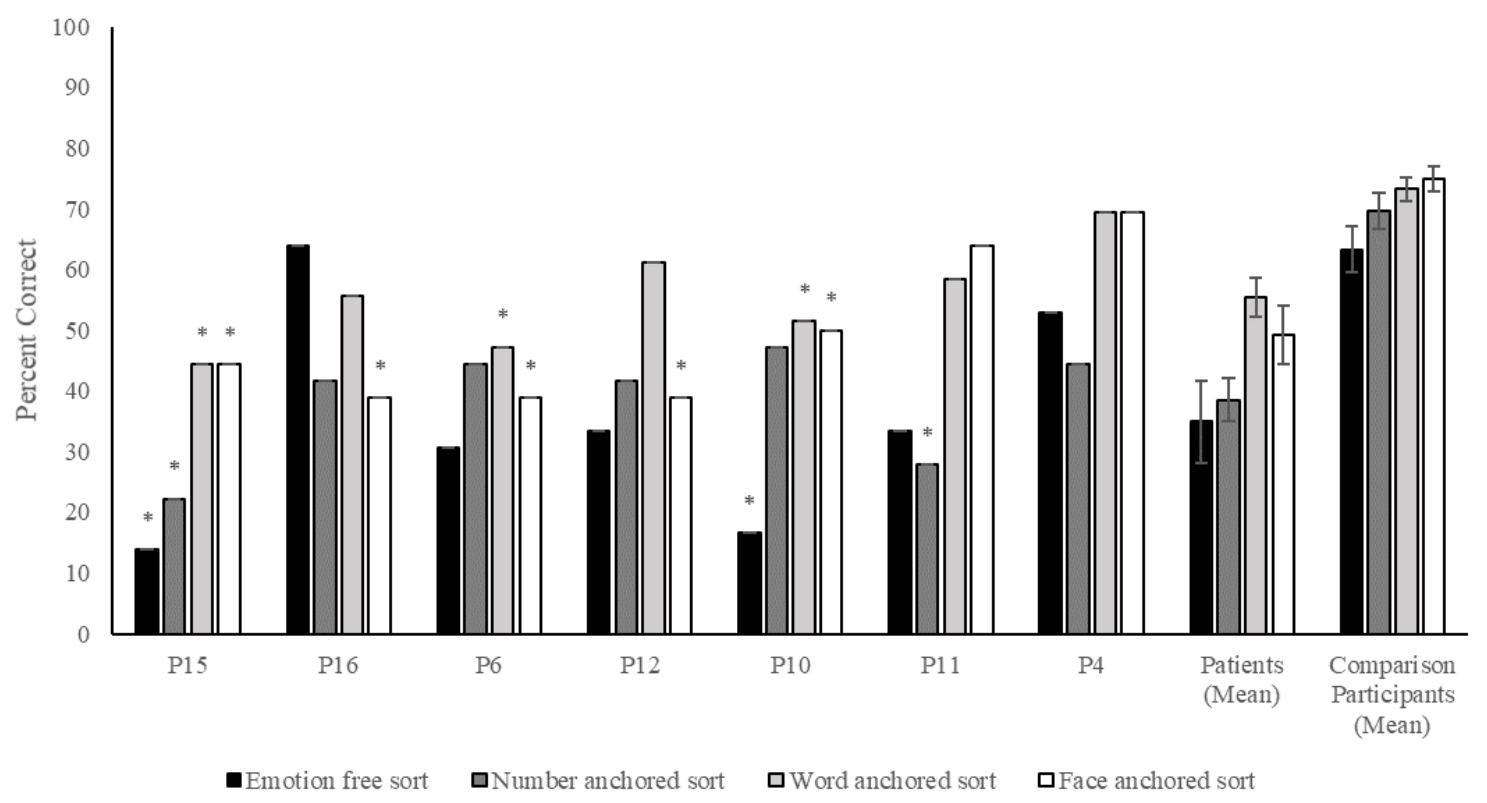

Figure 3: Total percent correct in the Study 1 emotion free sort, number anchored sort, word anchored sort, and face anchored sort tasks, for each individual patient, and the mean percent correct of patients and comparison participants. Patients are ordered (left to right) 
from the most to least semantically impaired, based on their semantic control composite score. Errors bars created using standard error of the mean. * = impaired performance based on Singlims analysis.

Patients not only showed lower overall accuracy but were more likely to make certain types of errors than comparison participants. As seen in Supplementary Table 5, SA patients were most likely to make an abnormal number of negative within-valence errors, relative to comparison participants. This resembled the SD patients in Lindquist et al. (2014; see Supplementary Table 6) and follows from the constructionist hypothesis that semantic knowledge is especially useful for disambiguating between same-valence faces (Lindquist et al., 2014). The SA patients also frequently showed abnormally high rates of neutral faces sorted into negative piles, and other across-valence mistakes. They appeared to be at least as impaired as the SD patients on emotion sorting: in the free sort task, although there was substantial variation in performance, SA patients had an average accuracy of $35 \%$, ranging between 14\% and 64\% (while the three SD patients in Lindquist et al., 2014, presented with $38 \%, 56 \%$, and $61 \%$ accuracy on this task).

Mixed effects logistic regressions for the accuracy data are reported in Table 1. For each of the three models, examining the impact of a type of constraining information (numbers, word labels, emotional faces) relative to performance in the free sort task, likelihood ratio tests revealed a significant contribution of group, with comparison participants more likely to produce a correct response than patients. A significant contribution of task was found for both the number and word anchored models, suggesting that these more constrained tasks increased the likelihood of correct responses, relative to free sort, while the task effect for the face anchored model approached significance. A significant interaction between task and group was observed for the word anchored model. As seen in Figure 3, this reflected greater facilitation of performance in SA patients than comparison participants from the provision of word anchors, compared with free sort, reflected by mean differences in accuracy between these tasks (patients: 20.4\%, comparison participants: 9.9\%). 
Table 1

Output for Study 1 mixed effects logistic regressions used to observe effects of group and task on the odds of sorting a given stimulus correctly.

\begin{tabular}{|c|c|c|c|c|}
\hline Variable & Estimate & Lower 95\% CI & Upper $95 \%$ CI & Likelihood Ratio Test \\
\hline \multicolumn{5}{|c|}{ Free sort vs number anchored sort } \\
\hline Intercept & -0.15 & -0.59 & 0.29 & - \\
\hline Task & -0.33 & -0.63 & -0.04 & $\chi(1)=4.90, p=.027 *$ \\
\hline Group & 1.08 & 0.60 & 1.57 & $\chi(1)=15.58, p<.001 *$ \\
\hline Task*Group & 0.27 & -0.06 & 0.60 & $\chi(1)=2.61, p=.106$ \\
\hline \multicolumn{5}{|c|}{ Free sort vs word anchored sort } \\
\hline Intercept & -.38 & -0.82 & 0.06 & - \\
\hline Task & 0.58 & 0.29 & 0.88 & $\chi(1)=15.54, p<.001 *$ \\
\hline Group & 1.25 & 0.76 & 1.74 & $\chi(1)=19.44, p<.001 *$ \\
\hline Task*Group & -0.37 & -0.70 & -0.04 & $\chi(1)=4.89, p=.027 *$ \\
\hline \multicolumn{5}{|c|}{ Free sort vs face anchored sort } \\
\hline Intercept & -0.30 & -0.74 & 0.14 & - \\
\hline Task & 0.27 & -0.02 & 0.56 & $\chi(1)=3.24, p=.072$ \\
\hline Group & 1.14 & 0.65 & 1.63 & $\chi(1)=16.75, p<.001 *$ \\
\hline Task*Group & 0.07 & -0.26 & 0.40 & $\chi(1)=0.18, p=.669$ \\
\hline
\end{tabular}

Note: * reflects significance at the .05 threshold. $\mathrm{CI}=$ confidence interval. Models run in $\mathrm{R}$ using lme4 package (version 1.1-25; Bates et al., 2015). As these are logistic models, estimate coefficients reflect log transformation of odds ratios (Larsen et al., 2000).

In summary, Study 1 found that SA patients, who presented with the hallmarks of deregulated retrieval, were at least as impaired as SD patients (with degraded semantic knowledge) at sorting emotional facial emotion portrayals. Patients made similar errors to those reported previously in SD, in which different within-valence emotions are confused. Patients with SA also benefitted to a greater extent than healthy comparison participants from emotion word anchors relative to free sort - presumably because these labels increased the accessibility of semantic information about different emotions in the context of deregulated retrieval.

\section{Study 2: Emotion cueing}

Study 1 provided evidence that impairments in semantic control can interfere with emotion categorization in patients with SA. Discrimination of facial portrayals of emotion may require inhibition of conceptually-similar emotional categories, particularly for states 
with prototypically high conceptual and perceptual similarity, such as anger and sadness. This conflict may result in misidentification of emotional portrayals in SA patients with deregulated semantic retrieval, as accuracy is impeded both by competition from superficial visual similarities and overgeneralisation of an overarching category (e.g., negative affect). These difficulties could be ameliorated in SA patients by providing conceptual cues which make the target information more accessible, consistent with evidence that multimodal cues facilitate access to conceptual information in SA (Jefferies et al., 2008; Lanzoni et al., 2019; Noonan et al., 2010). Equally, miscueing irrelevant information may increase selection demands, and therefore impede accurate emotion categorization (Noonan et al., 2010). Study 2 established whether the retrieval of emotional categories in SA was influenced by auditory cues and miscues to emotion categories (i.e., prosody), in an alternative forced-choice design. Cues were predicted to reduce semantic control demands by constraining interpretation of the subsequent portrayal. Within-valence miscues were predicted to increase semantic control demands by requiring participants to inhibit irrelevant but conceptually similar information. Across-valence miscues were not predicted to affect semantic control demands, as the miscued information is conceptually dissimilar to the target emotion category.

\subsection{Method}

\subsubsection{Participants}

A subset of the participants from Study 1 participated in Study 2. This included six patients (three female) with a mean age of $60.0(\mathrm{SD}=6.3)$, a mean age of leaving education of $18.8(\mathrm{SD}=4.0)$ and a mean of 11.6 years $(\mathrm{SD}=6.0)$ since their most recent stroke. The sample also included 15 healthy comparison participants (11 female) with a mean age of 65.1 $(\mathrm{SD}=7.1)$, and a mean age of leaving education of 20.5 ( $\mathrm{SD}=2.2)$. Patients and comparison participants did not significantly differ on age: $\mathrm{t}(19)=-1.5, p=.140$, or on age when leaving education: $\mathrm{t}(19)=-1.3, p=.223$.

\subsubsection{Design}

Study 2 required participants to match individually presented facial portrayals to one of three emotion labels: 'angry', 'happy', and 'sad'. Trials were preceded by either a 'cue'/'miscue' period or a 'no cue' period. A repeated measures design was used, with all participants completing the task across the four conditions of cue, no cue, miscue within- 
valence, and miscue across-valence. Facial stimuli and prosody cues were restricted to the emotions of anger, happiness, and sadness. On cue trials, facial stimuli were preceded by an auditory cue which was congruent in emotion with the face displayed (e.g., a happy voice preceding a happy face). On miscue within-valence trials, facial stimuli were preceded by an incongruent prosody cue of the same valence, but of a different emotion (e.g., an angry voice preceding a sad face). On miscue across-valence trials, facial stimuli were preceded by an incongruent prosody cue of the opposite valence (e.g., a happy voice preceding an angry face). Prosody cues were recordings of "babbling" (ba-ba-ba, without semantic content), spoken in different tones of voice associated with specific emotions. The study was conducted over two sessions, with 66 trials in each session, and 132 trials overall. Session order was counterbalanced across participants. Trials were split over 36 cue, 36 no cue, 36 miscue across-valence, and 24 miscue within-valence trials. Fewer miscue within-valence trials were included as it was not possible to provide within-valence miscues for happy portrayals. Each condition and probe emotion were equally represented across the two sessions.

\subsubsection{Stimuli}

As with Study 1, stimuli were taken from the IASLab Face Set (https://www.affective-science.org/face-set.shtml). A full list of stimuli used (by identity code) can be seen in Supplementary Table 4. Facial muscle portrayals of the English language emotion categories anger, happiness, and sadness were obtained for 12 identities (half male, half female), resulting in 36 stimuli overall. Prosody cues were taken from the Aprosodia Battery (Ross et al., 1997). These were recordings of actors saying "ba-ba-ba-baba" with either angry, happy, or sad prosody. These cues were seven, eight, and six syllables

long, respectively. The same single angry, happy, and sad prosody cues were used throughout the experiment.

\subsubsection{Procedure}

Study 2 was administered online due to COVID-19 social distancing restrictions. Stimuli were presented using PsychoPy3 (Peirce et al., 2019). Before beginning, the researcher called the participant over Zoom (Zoom Video Communications Inc., 2016). Both the participant's and researcher's video were turned off, to prevent them obscuring the stimuli. The researcher shared their screen and computer sound with the participant and gave them remote control of their cursor, which remained visible on the screen. Figure 4 provides 
a summary of the Study 2 stimuli and procedure, including examples of stimuli.

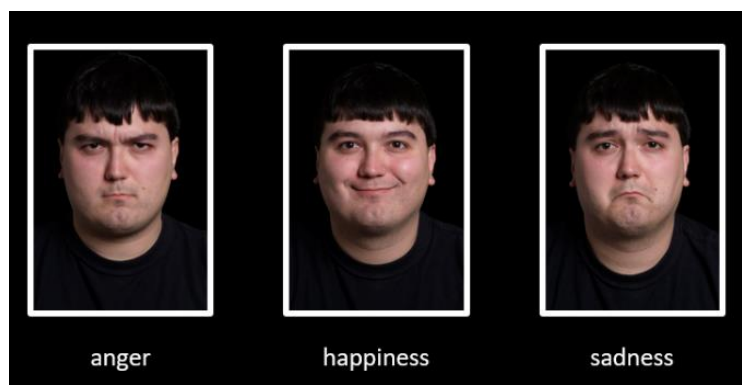

(1)

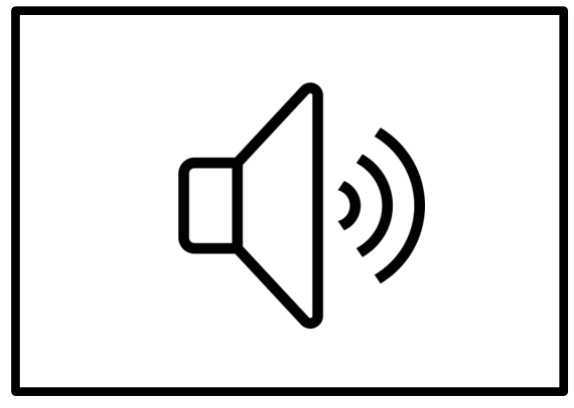

(2)

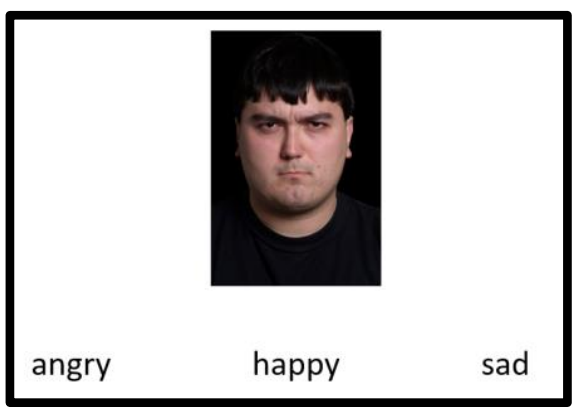

(4)

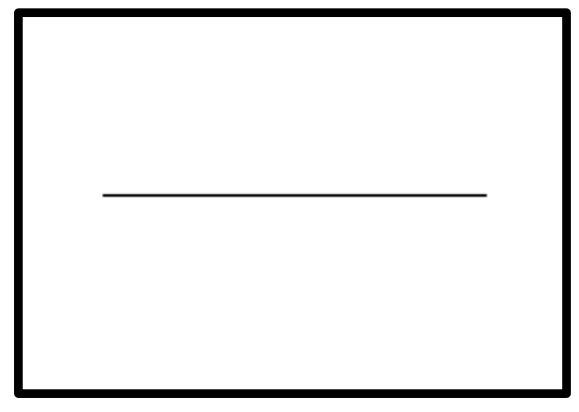

(3)

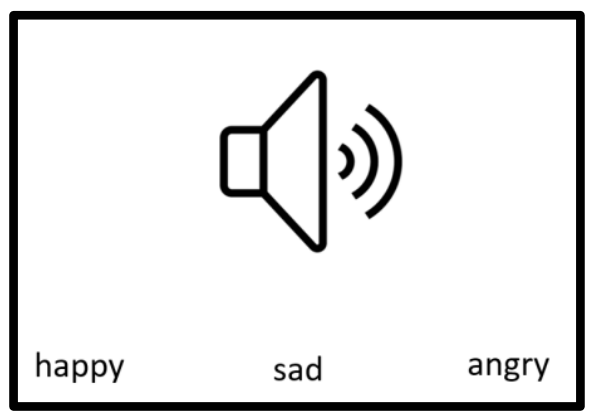

(5)

Figure 4: A summary of the Study 2 stimuli and procedure, including (1) examples of stimuli, graphics used during the (2) 'cue' or 'miscue' phase and the (3) 'no cue' phase, and presentation of stimuli and response options during (4) the experimental decision phase and (5) the 'prosody identification' trials which followed completion of the study.

Participants were told: "On each trial, you will be presented with one picture, with three words underneath it. Each word will correspond with an emotion that the person in the picture could be feeling. It will be your job to indicate which emotion you think the person in the photo is feeling. To do this, move the cursor over the word you want to select.". Prior to each session, participants were presented with five practice trials, which included feedback. 
Each participant demonstrated an understanding of the task instructions and verified that they could clearly hear the audio cues during these trials.

Each trial was preceded by either a cue/miscue or a no cue phase. For cue/miscue trials, a graphic of a loudspeaker appeared on the screen while the audio played for approximately 3.5 seconds. In no cue trials, a horizontal black line appeared in place of the loudspeaker, for a silent period of 3.5 seconds. In the decision phase of the task, a face appeared in the middle of the screen with three emotion labels beneath it: 'angry', 'happy', and 'sad'. The position of these labels, and of the target label, was counterbalanced across trials. Due to impaired reading ability in some patients, these labels were read aloud from left to right on each trial for patients, but not for comparison participants. To indicate their response, the participants moved the cursor over the word they wished to choose, and the researcher keyed in their response. This was viewed as the closest analogue to participants pointing at the screen (the typical method employed in our in-person testing). Each trial was followed by a 1.5 second fixation cross. All trials were presented in a randomised order. The position of the response options was moved by 150 pixels in the horizontal direction for each alternating trial, which ensured that the participant always needed to move the cursor to indicate their response, even in cases where the position of the correct response was the same over subsequent trials. Participants were offered a break at the half-way point of each session. After the completion of the second session, participants completed a 'prosody identification' task, in which they were presented with the three audio cues in isolation and asked to match them to their respective emotion label.

\subsubsection{Data Analysis}

For each participant, mean accuracy (percentage correct) and response time (seconds) in each condition were recorded. Each patient was classified as being either impaired or not impaired in their overall accuracy for each condition. As in Study 1, this was done using Singlims (Crawford et al., 2010). Again, one-tailed p-values were interpreted against a significance threshold of .05. As in Study 1, a mixed effects logistic regression was performed in R (R Core Team, 2020), to compare accuracy across groups and on each of the cue conditions (cues, across-valence miscues, within-valence miscues) relative to the no cue condition. While Study 1 employed serially presented discrete tasks, the current analysis compared performance across conditions within a single experiment within one omnibus model. Group and condition were entered as dichotomous fixed factors, and participant identity as a random factor. These factors were used to predict the likelihood of a correct 
response for each individual trial, coded as either correct or incorrect. The model was fitted using maximum likelihood, based on Gaussian Hermite approximation. The model was run using the lme4 package (version 1.1-25; Bates et al., 2015). As this is a logistic model, estimate coefficients reflect log transformation of odds ratios (Larsen et al., 2000).

Likelihood ratio tests were used to test for the significance of effects or interactions of group and condition, by statistically comparing the overall model to nested versions with specific effects removed. Participants' mean response time across conditions is presented in a bar graph in Supplementary Figure 1 and analysed using a mixed effects linear regression in Supplementary Table 8, followed by pairwise contrasts in Supplementary Table 9. Due to the data collection occurring over Zoom, this response time analysis should be interpreted cautiously. All data for Study 2 are publicly available on Mendeley Data (https://data.mendeley.com/datasets/zwxxscny6x/3).

\subsection{Results}

Each patient's percentage accuracy for each condition, as well as group averages for patients and comparison participants, can be seen in Figure 5. As in Study 1, patients in Figure 5 are ordered from most to least impaired (from left to right). As determined by Singlims analysis, four of the six patients were impaired for at least one condition, relative to comparison participants (with normal performance overall for the least and third least semantically impaired patients). All participants performed with $100 \%$ accuracy when categorizing happy portrayals, regardless of condition, and angry and sad faces were never erroneously labelled as happy. All errors were within-valence, involving confusion of angry and sad portrayals. 


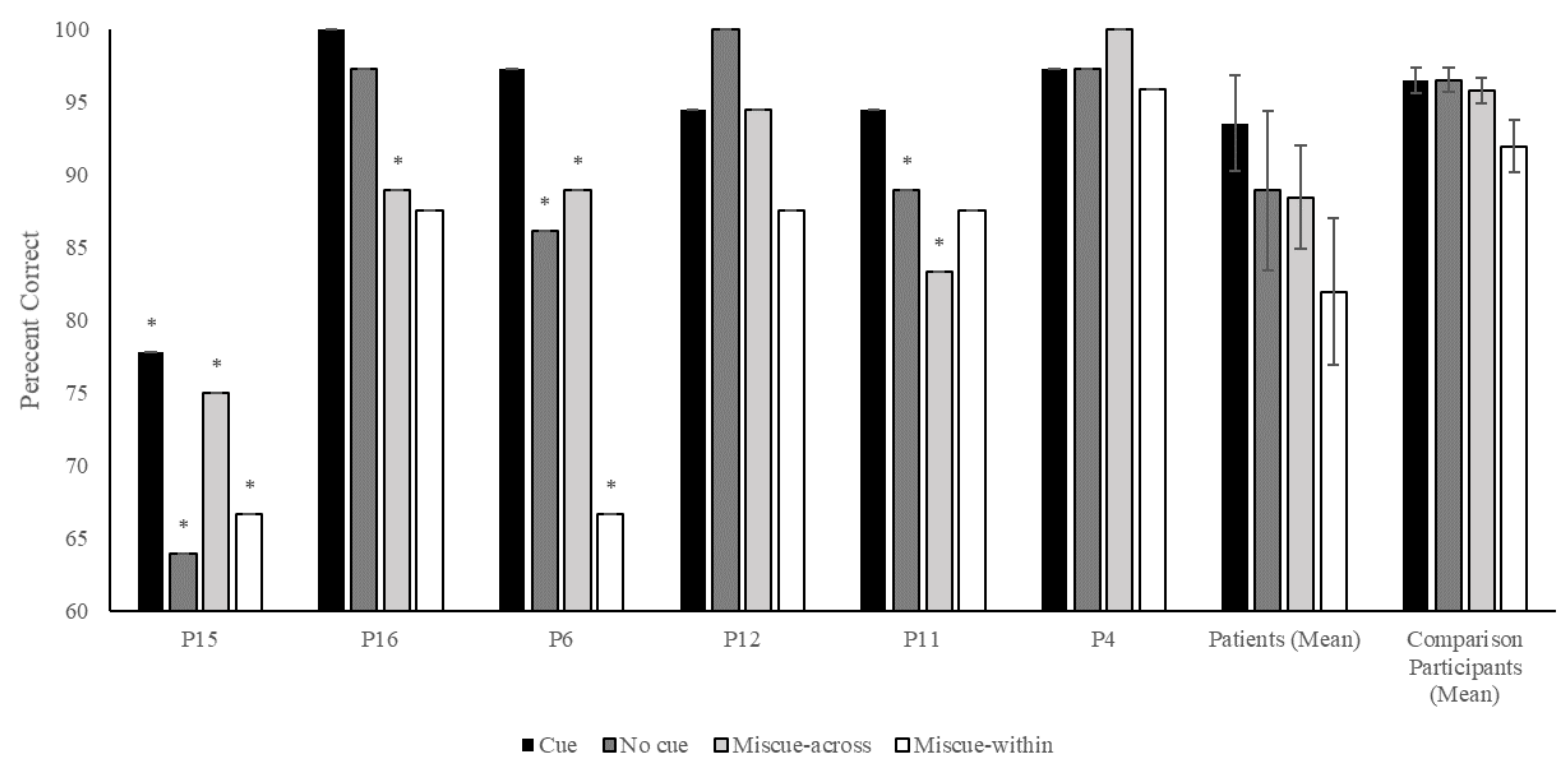

Figure 5: Total percent correct in the cue, no cue, miscue across-valence, and miscue withinvalence conditions, for each individual patient and group means. Patients are ordered (left to right) from the most to least semantically impaired, based on their semantic control composite score. Errors bars reflect standard error of the mean. ${ }^{*}=$ impaired performance based on Singlims analysis.

The results of the mixed effects logistic regression for accuracy are reported in Table 2. The model revealed a significant contribution of group, with comparison participants more likely to produce a correct response than patients. A significant effect of condition was found. The group by condition interaction was non-significant. To parse the observed condition main effect, we observed pairwise post-hoc comparisons using the emmeans package (Lenth, 2020) in R, with the Tukey HSD method for multiple comparisons applied. We observed the difference between each combination of conditions, reported in Table 3. Contrasts were split by group as we were specifically interested in effects of cueing in SA. Patients had a significantly higher probability of a correct response during cue than miscue within-valence trials. This suggests a difference in performance between the trials with the highest and lowest semantic control demands. Comparison participants showed this same effect, but also demonstrated diminished probability of a correct response in miscue within-valence trials relative to the no cue baseline. Neither group showed significant difference between cue and no cue trials. 
Table 2

Output for the Study 2 mixed effects logistic regression observing effects of group and condition on the odds of responding correctly to a given trial.

\begin{tabular}{lcccc}
\hline Variable & Estimate & Lower 95\% CI & Upper 95\% CI & Likelihood Ratio Test \\
\hline Intercept & 2.33 & 1.57 & 3.09 & - \\
Group & 1.21 & 0.24 & 2.18 & $\chi(1)=5.57, p=.018^{*}$ \\
Condition & - & - & - & $\chi(3)=12.3, p=.006^{*}$ \\
Group by Condition & - & - & - & $\chi(3)=1.75, p=.626$ \\
\hline
\end{tabular}

Note: * reflects significance at the .05 threshold. $\mathrm{CI}=$ confidence interval. Model was run in $\mathrm{R}$ using lme4 package (version 1.1-25; Bates et al., 2015). As this is a logistic model, estimate coefficients reflect log transformation of odds ratios (Larsen et al., 2000). Overall condition effect and group by condition interaction do not include an estimate value, as these effects are not provided by the overall model. The respective likelihood ratio test results were obtained by comparing the full model to nested versions in which all condition main effects or interactions were removed.

Table 3

Post-hoc pairwise contrasts of the Study 2 accuracy mixed effects logistic regression, comparing the likelihood of a correct response across conditions within the patient and comparison groups.

\begin{tabular}{lcc}
\hline Contrast & Patients & Comparison Participants \\
\hline No cue - cue & $\mathrm{OR}=0.54, p=.308$ & $\mathrm{OR}=1, \mathrm{p}=1$ \\
No cue - miscue across-valence & $\mathrm{OR}=1.05, p=.999$ & $\mathrm{OR}=1.22, p=.921$ \\
No cue - miscue within-valence & $\mathrm{OR}=1.85, p=.216$ & $\mathrm{OR}=2.46, p=.018^{*}$ \\
Cue - miscue across-valence & $\mathrm{OR}=1.96, p=.237$ & $\mathrm{OR}=1.22, p=.921$ \\
Cue - miscue within-valence & $\mathrm{OR}=3.46, p=.004^{*}$ & $\mathrm{OR}=2.46, p=.018^{*}$ \\
Miscue across-valence - miscue within-valence & $\mathrm{OR}=1.76, p=.279$ & $\mathrm{OR}=2.01, p=.080$ \\
\hline
\end{tabular}

Note: * reflects significance at the .05 threshold. P-values were corrected using the Tukey HSD method for multiple comparisons. Contrasts were run using the emmeans package (Lenth, 2020) in R. OR = odds ratio.

When tested on prosody identification, all comparison participants accurately labelled each cue. Three patients made mistakes, with P16 labelling the happy cue as "angry", P6 labelling the angry cue as "sad", and P15 switching the labels for angry and sad cues. This may suggest diminished ability to accurately perceive emotion from prosody cues (i.e., impaired emotion perception across modalities), as suggested by prior studies of left hemisphere stroke patients (Leung et al., 2017). Despite this, all three of these patients 
performed better on trials with cues than on no cue and miscue trials (in two cases, by a margin of over $10 \%$, see Figure 5). This implicit sensitivity to emotion cues, even when not explicitly identified, is consistent with deregulated semantic access in SA.

\section{$\underline{\text { Discussion }}$}

The current study assessed the ability to perceive and categorize discrete facial emotion portrayals in SA patients with multimodal impairment in controlled semantic retrieval following left hemisphere stroke. Constructionist models of emotion argue for a necessary role of conceptual knowledge in guiding emotion perception, insofar as conceptual knowledge helps to transform otherwise ambiguous facial muscle movements into perceptions of discrete emotion categories (Barrett et al., 2007; Lindquist et al., 2015; 2016). Accordingly, there is evidence of impaired facial emotion perception when access to emotion concepts is temporarily impaired in healthy comparison participants due to semantic satiation (Gendron et al., 2012; Lindquist et al., 2006). Progressive loss of heteromodal concepts in semantic dementia similarly results in impaired facial emotion perception (Lindquist et al., 2014). This study builds on these findings to demonstrate that deficits of controlled semantic retrieval in patients with SA are similarly associated with impaired emotion perception.

In Study 1, we adapted a paradigm from Lindquist et al. (2014), previously used with SD patients. This task required patients to sort pictures of facial emotion portrayals into piles corresponding to discrete emotional states, with minimal task instruction, and with numbered, word, and face anchors. As predicted, comparison participants consistently outperformed SA patients, with all but one patient being impaired on at least one sorting task. Impairments in this group were at least as severe as those previously observed in SD. Moreover, the SA group did not show impairment on a control task in which they sorted faces according to identity, suggesting that general deficits in sorting behaviour are unlikely to explain their difficulties in sorting portrayals of categorical emotional states. Constraining the intended number of discrete emotion portrayals with number anchors improved accuracy across the sample. Benefits of face anchors were marginal but did not reach significance. While both groups benefited from word anchors, these benefits were larger for SA patients than comparison participants. These results are consistent with the view that semantic control is needed to resolve competition between 
conceptually similar emotion categories, and to access categorical information in the face of ambiguous input. Introducing relevant conceptual information (word anchors) may have ameliorated patients' deficits by reducing this ambiguity.

Study 2 required participants to match facial emotion portrayals to emotion labels in a three alternative forced choice design. Trials were preceded by affective prosody cues or miscues, presented auditorily, alluding to the correct or incorrect interpretation of the subsequent portrayal of emotion. These cues were compared to a no cue control condition. In line with our hypotheses, SA patients were consistently impaired relative to the comparison group. Patients presented with significantly higher accuracy on cue trials when compared to the most challenging within-valence miscue trials. Comparison participants showed this same pattern, but also presented with lower accuracy on withinvalence miscue trials when compared to the no cue baseline. As previously observed for other modalities (e.g., Lanzoni et al., 2019), semantically-related miscues may have resulted in more errors overall because they increased the competition from conceptuallyrelated distractor emotions. The lack of a difference between no cue and miscue-within valence trials in SA might reflect the way that some patients (e.g., P15, P11) performed poorly on both conditions, while comparison participants frequently performed near ceiling-level in the absence of cues, but made errors following within-valence miscues. This null result might also reflect high variability across conditions in patients, relative to the more homogeneous performance seen in the comparison participants. No effect of the less challenging across-valence miscues was observed in either group. The lack of a condition by group interaction suggests that effects of miscuing were equivalent for patients and comparison participants. Again, this study implicates controlled semantic retrieval in emotion processing.

Overall, these results are consistent with the constructionist claim that emotion perception is reliant on conceptual representations of emotional states (Lindquist et al., 2015). Semantic storage deficits (i.e., in SD) are already known to impair emotion discrimination and categorization (Kumfor et al., 2018; Miller et al., 2012; Lindquist et al., 2014; Rosen et al., 2002); this study extends these observations to SA patients thought to have deregulated semantic cognition following left hemisphere stroke. If perception of emotion from facial portrayals in non-verbal and automatic (Tracy \& Robins, 2008) or if activation of emotion concepts is automatic upon perceiving relevant emotion portrayals (Preston, 2007), semantic control impairments, which affect the retrieval of concepts 
under ambiguous conditions, might not be expected to interfere with emotion categorization. Our results do not support this account, since SA patients have deficits of emotion perception which are at least equivalent to those seen following storage deficits. This finding builds on the constructionist account of emotion, in suggesting that, as well as intact representations, semantic control is required for effective emotion discrimination. Emotion categorization may not be entirely automatic and can be disrupted when there is ambiguity or competition. Under this revised framework, emotion perception can be viewed as reliant on the successful resolution of ambiguity and selection among competing conceptual representations, as has been observed for semantic decisions in other domains (Noonan et al., 2010).

A comparison of the SA patients in this study with the SD sample in Lindquist et al. (2014) supports the hypothesis, stemming from the CSC framework, that SA patients show greater impairment when task demands are high (Jefferies, 2013). Errors in the original sample of SD patients were largely constrained to within-valence mistakes. In the current SA sample, within-valence errors were still the most common, but patients also produced across-valence errors, often confusing negative and neutral faces. Across the four tasks, $10 \%$ of piles made by patients were found to include faces with one predominant identity (i.e., $50 \%+$ pictures in a pile had the same identity). This suggests that some errors in the SA group may have been driven by the perceptual similarities between faces with the same identity, which may have interfered with judgements about emotion. Moreover, of the two SD patients from Lindquist et al. (2014) who had completed the constrained conditions, both appeared to benefit considerably from the presence of face anchors, but not word anchors. The inverse relationship was found in the current sample, with SA patients benefiting from word anchors but not face anchors, perhaps because the words were stronger cues to distinct categories of emotion. While both picture and word cues have been effective in earlier studies of semantic cognition in SA (e.g., Corbett et al., 2011; Jefferies et al., 2008) the face anchors provided similar information to the target pictures, as opposed to an explicit guide to the way these stimuli could be categorised. These findings conceptually replicate evidence that individuals with alexithymia, a subclinical trait associated with difficulty labelling and describing emotions, benefited more from the presence of word primes than face primes in a repetition priming task (Nook et al., 2015). 
Contrary to prior research (e.g., Lanzoni et al., 2019; Noonan et al., 2010), Study 2 provides limited evidence of the effectiveness of cues in facilitating access to relevant information. Patients' accuracy significantly increased in the cue relative to the withinvalence miscue trials, suggesting a difference across manipulations which maximally vary in their semantic control demands, but there was no effect of cues on accuracy relative to the no cue baseline. This may in part be due to the limited patient sample size in the current study, or due to the fact that several patients performed near ceiling-level in the baseline no cue condition, leaving limited opportunities for cue-induced improvement. Deleterious effects of within-valence miscues across the sample are consistent with prior evidence that task-irrelevant miscues impair semantic judgements in SA by increasing the need to internally constrain retrieval (Noonan et al., 2010). These miscues required participants to inhibit emotion concepts which were irrelevant but conceptually similar to the target emotion. The inhibition of competing conceptual information is a key feature of semantic control (Gray, 2020; Hoffman, 2018), and lower accuracy under this condition was therefore anticipated. In comparison, across-valence miscues that were less conceptually similar, did not affect participants' accuracy. We observed equivalent effects of within-valence miscues for SA patients and control participants, consistent with evidence that priming irrelevant emotion labels can interfere with healthy adults' identification of discrete facial emotion portrayals (Fugate et al., 2018; Lindquist et al., 2006). This effect was expected to be larger in patients than in healthy controls; subsequent work employing a larger sample of SA patients is needed to establish if the effect size is equivalent for these two groups.

\subsection{Limitations}

There are some limitations stemming from the remote testing that we employed as a result of the COVID-19 pandemic. Under these restrictions it was not possible to obtain full structural scans of four patients in the current sample, limiting our ability to associate impaired emotion perception with lesion site and therefore to make precise neuroanatomical conclusions. Furthermore, remote testing directly led to the exclusion of more severely-impaired SA patients who were unable to engage with the technological demands of the tasks. Communication was also more challenging under these conditions, and the instructions of Study 1 were complex - yet the patient group showed no impairment in the control task which involved sorting by identity. They were similarly impaired in Study 2, which probed emotion categorization with far simpler task demands. 
Viewing conditions during online testing were also more challenging than in face-to-face testing. Controls showed similar performance on the emotion free sort condition here (63.3\% correct) and in the original paradigm (70.0\% correct; Lindquist et al., 2014). However, there was a difference on the identity sort task: all SD patients and controls in Lindquist et al. performed perfectly, while in our Study 1, three patients and five controls made errors. Participants who made errors on this task did not show poorer performance on the emotion sort task (see section 3.2.1); consequently, these errors may have reflected suboptimal viewing conditions and the challenges of computer-assisted testing.

In common with other studies of SA, the patients in the current sample were not solely impaired on background tests of semantic control and did present with some nonverbal and non-semantic deficits in executive function and visuospatial processing (Thompson et al., 2018). This is unsurprising given that the semantic control network is adjacent to left hemisphere components of the bilateral multiple-demand network, which supports executive control across domains (Gao et al., 2021; Wang et al., 2020). As a result, the two networks are often damaged together by stroke, although deficits of semantic control are likely to be more severe following left-hemisphere stroke (Thompson et al., 2016), since this network is strongly left-lateralised (Gao et al., 2021; Gonzalez Alam et al., 2019). A more general cognitive impairment was particularly marked in background neuropsychological testing for P15, who also presented with the poorest overall performance in both Studies 1 and 2. It is worth noting that P15 did present with preserved performance on some demanding non-semantic tasks, including the Test of Everyday Attention, and the Trail Making Test B. Furthermore, some of the easier tasks that P15 was below the official cut-off for (e.g. Trail Making Test A, wordpicture matching) nevertheless elicited very few errors. Regardless, we cannot provide definitive evidence that non-semantic impairments in the sample did not contribute to poor emotion categorization, even though the SA group were not impaired on a control identity sort task, relative to the comparison group.

Comparisons between the current SA patients and the SD patients of Lindquist et al. (2014) should be made cautiously given the disparities in the testing environment. The current findings allow only for qualitative comparison between these groups. A recent study of 16 SD patients provided evidence of correlations between category fluency performance, facial emotion recognition ability, and conceptual and taxonomic 
knowledge about emotion (Bertoux et al., 2020). Future research with a similarly large sample of both SD and SA patients could be helpful in understanding the effects of both the nature and severity of semantic impairment on emotion categorization deficits. It would also be interesting to relate deficits of emotional recognition in SA to emotional difficulties commonly seen following stroke, including depression and anxiety (Døli et al., 2017) and alexithymia (Hobson et al., 2020). However, the paradigms employed here probed semantic valence (i.e., knowledge about affective qualities, e.g., knowing that flowers are positively valenced), rather than experiential affect (e.g., feeling delight; Itkes et al., 2019; Itkes \& Kron, 2019), and as such do not address this issue directly.

Recently, Sauter (2018) argued that language-based models of emotion processing may place too much weight on the assumption of a direct link between lexical, conceptual, and perceptual processing of emotion, and that "inferences cannot be drawn directly from findings in one domain (e.g., emotion words) to another (e.g., emotion perception, emotion production, or emotion experience)" (p. 112). While the current studies used verbal and auditory prompts to facilitate access to emotion concepts, our analysis was confined to the perception of facial emotion portrayals. These findings therefore cannot be directly applied, for example, to the ability to sort words by emotion. As semantic control impairments in SA are multimodal (Jefferies, 2013), future research could test the prediction that emotion processing impairments extend across modalities (see Osborne-Crowley et al., 2020 for an example from patients with traumatic brain injury).

\subsection{Conclusion}

The current study provides evidence that impaired controlled semantic retrieval in SA disrupts the perception of discrete emotion categories for portrayals of facial emotion, and that manipulating access to relevant conceptual information can affect emotion perception, even in the absence of impaired semantic or language processing. These findings lend support to the constructionist model of emotion in suggesting a foundational role of conceptual knowledge in supporting emotion perception. Access to emotion category representations appears to involve semantic control processes, which are required to resolve ambiguity and to select representations among competing distractors. 


\section{Acknowledgements}

We are eternally grateful to the patients, their carers, partners, and families, and the comparison participants for the time they have given, and continue to give, to support our research. We would also like to thank Marcus Glennon and Anna Clegg for their assistance with data collection, and Sarah Knight for her guidance in the analysis used. EJ was funded by an ERC Consolidator grant (FLEXSEM - 771863).

Competing Interests Statement

The authors have no competing interests to disclose.

Data Availability Statement

All data for the current studies are publicly available on Mendeley Data (https://data.mendeley.com/datasets/zwxxscny6x/3). 


\section{References}

Avants B. B, Tustison, N. J., Song, G., Cook, P. A., Klein, A., \& Gee, J. C. (2011) A reproducible evaluation of ANTs similarity metric performance in brain image registration. NeuroImage, 54(3), 2033-44.

https://doi.org/10.1016/j.neuroimage.2010.09.025.

Aviezer, H., Hassin, R. R., Ryan, J., Grady, C., Susskind, J., Anderson, A., Moscovitch, M., \& Bentin, S. (2008). Angry, disgusted, or afraid?: Studies on the malleability of emotion perception. Psychological Science, 19(7), 724-731. https://doi.org/10.1111/j.1467-9280.2008.02148.x.

Badre, D., Poldrack, R. A., Paré-Blagoev, E. J., Insler, R. Z., \& Wagner, A. D. (2005). Dissociable controlled retrieval and generalized selection mechanisms in ventrolateral prefrontal cortex. Neuron, 47(6), 907-918. https://doi.org/10.1016/j.neuron.2005.07.023.

Barrett, L. F., Lindquist, K. A., \& Gendron, M. (2007). Language as context for the perception of emotion. Trends in Cognitive Science, 11(8), 327-332. https://doi.org/10.1016/j.tics.2007.06.003.

Bates, D., Mächler, M., Bolker, B., \& Walker, S. (2015). Fitting Linear Mixed-Effects Models Using lme4. Journal of Statistical Software, 67(1), 1-48. https://doi.org/10.18637/jss.v067.i01.

Bell, A., Fairbrother, M., \& Jones, K. (2019). Fixed and random effects models: Making an informed choice. Quality \& Quantity, 53, 1051-1074. https://doi.org/10.1007/s11135-018-0802-x. 
Bertoux, M., Duclos, H., Caillaud, M., Segobin, S., Merck, C., de La Sayette, V., Belliard, S., Desranges, B., Eustache, F., \& Laisney, M. (2020). When affect overlaps with concept: emotion recognition in semantic variant of primary progressive aphasia. Brain, 143, 3850-3864. https://doi.org/10.1093/brain/awaa313.

Bozeat, S., Gregory, C. A., Lambon Ralph, M. A., \& Hodges, J. R. (2000). Which neuropsychiatric and behavioural features distinguish frontal and temporal variants of frontotemporal dementia from Alzheimer's disease? Journal of Neurology, Neurosurgery, and Psychiatry, 69(2), 178-186. https://doi.org/10.1136/jnnp.69.2.178.

Bozeat, S., Lambon Ralph, M. A., Patterson, K., Garrard, P., \& Hodges, J. R. (2000). Nonverbal semantic impairment in semantic dementia. Neuropsychologia, 9, 1207-1215. https://doi.org/10.1016/s0028-3932(00)00034-8.

Brooks, J. A., \& Freeman, J. B. (2018). Conceptual knowledge predicts the representational structure of facial emotion perception. Nature Human Behaviour, 2, 581-591. https://doi.org/10.1038/s41562-018-0376-6.

Burgess, P. W., \& Shallice, T. (1997). The Hayling and Brixton Tests. Bury St Edmunds: Thames Valley Test Company.

Carroll, N., C., \& Young, A. W. (2005). Priming of emotion recognition. Quarterly Journal of Experimental Psychology, 58A(7), 1173-1197. https://doi.org/10.1080/02724980443000539.

Chapman, C. A., Hasan, O., Schulz, P. E., \& Martin, R. C. (2020). Evaluating the distinction between semantic knowledge and semantic access: Evidence from semantic dementia and comprehension-impaired stroke aphasia. Psychonomic Bulletin \& Review, 27, 607-639. https://doi.org/10.3758/s13423-019-01706-6. 
Chiou, R., Humphreys, G. F., Jung, J., \& Lambon Ralph, M. A. (2018). Controlled semantic cognition relies upon dynamic and flexible interactions between the executive 'semantic control' and hub-and-spoke 'semantic representation' systems. Cortex, 103, 100-116. https://doi.org/10.1016/j.cortex.2018.02.018.

Corbett, F., Jefferies, E., \& Lambon Ralph, M. A. (2011). Deregulated semantic cognition follows prefrontal and temporo-parietal damage: evidence from the impact of task constraint on nonverbal object use. Journal of Cognitive Neuroscience, 23(5), 11251135. https://doi.org/10.1162/jocn.2010.21539.

Crawford, J. R., Garthwaite, P. H., \& Porter, S. (2010). Point and interval estimates of effect sizes for the case-controls design in neuropsychology: Rationale, methods, implementations, and proposed reporting standards. Cognitive Neuropsychology, 27, 245-260. https://doi.org/10.1080/02643294.2010.513967.

Crawford, J. R., \& Howell, D. C. (1998). Comparing an individual's test score against norms derived from small samples. The Clinical Neuropsychologist, 12, 482-486. https://doi.org/10.1076/clin.12.4.482.7241.

Davey, J., Thompson, H. E., Hallam, G., Karapanagiotidis, T., Murphy, C., De Caso, I., Krieger-Redwood, K., Bernhardt, B. C., Smallwood, J., \& Jefferies, E. (2016). Exploring the role of the posterior middle temporal gyrus in semantic cognition: Integration of anterior temporal lobe with executive processes. NeuroImage, 137, 165-177. https://doi.org/10.1016/j.neuroimage.2016.05.051.

Diachek, E., Blank, I., Siegelman, M., Affourtit, J., \& Fedorenko, E. (2020). The domaingeneral multiple demand (MD) network does not support core aspects of language comprehension: a large-scale fMRI investigation. The Journal of Neuroscience, 40(23), 4536-4550. https://doi.org/10.1523/JNEUROSCI.2036-19.2020. 
Døli, H., Helland, T., \& Helland, W. A. (2017). Self-reported symptoms of anxiety and depression in chronic stroke patients with and without aphasia. Aphasiology, 31(12), 1392-1409. https://doi.org/10.1080/02687038.2017.1280595.

Doyle, C. M., Gendron, M., \& Lindquist, K. A. (2021). Language is a unique context for emotion perception. Affective Science, 2, 171-177. https://doi.org/10.1016/j.tics.2007.06.003.

Fugate, J. M. B., Gendron, M., Nakashima, S. F., \& Barrett, L. F. (2018). Emotion words: adding face value. Emotion, 18(5), 693-706. https://doi.org/10.1037/emo0000330.

Gao, Z., Zheng, L., Chiou, R., Gouws, A., Krieger-Redwood, K., Wang, X., Varga, D., Lambon Ralph, M. A., Smallwood, J., \& Jefferies, E. (2021). Distinct and common neural coding of semantic and non-semantic control demands. NeuroImage, 236, 118230. https://doi.org/10.1016/j.neuroimage.2021.118230.

Gendron, M., Lindquist, K. A., Barsalou, L., \& Barrett, L. F. (2012). Emotion words shape emotion percepts. Emotion, 12(2), 314-325. https://doi.org/10.1037/a0026007.

Gonzalez Alam, T. R. D. J., Karapanagiotidis, T., Smallwood, J., \& Jefferies, E. (2019). Degrees of lateralisation in semantic cognition: Evidence from intrinsic connectivity. NeuroImage, 202, 116089. https://doi.org/10.1016/j.neuroimage.2019.116089.

Gray, T. (2020). The relationship between language control, semantic control and nonverbal control. Behavioral Sciences, 10(11), 169. https://doi.org/10.3390/bs10110169.

Hallam, G. P., Whitney, C., Hymers, M., Gouws, A. D., \& Jefferies, E. (2016). Charting the effects of TMS with fMRI: Modulation of cortical recruitment within the distributed network supporting semantic control. Neuropsychologia, 93, 40-52. https://doi.org/10.1016/j.neuropsychologia.2016.09.012. 
Head, H. (1926). Aphasia and kindred disorders of speech (Vol. II). New York: Cambridge University Press.

Hobson, H., Chiu, E. G., Ravenscroft, C., Partridge, K., Bird, G., \& Demeyere, N. (2020). The association between communication impairments and acquired alexithymia in chronic stroke patients. Experimental Neuropsychology, 42(5), 495-504. https://doi.org/10.1080/13803395.2020.1770703.

Hodges, J. R., \& Patterson, K. (2007). Semantic dementia: a unique clinicopathological syndrome. The Lancet Neurology, 6(11), 1004-1014. https://doi.org/10.1016/S14744422(07)70266-1.

Hoemann, K., Xu, F., \& Barrett, L. F. (2019). Emotion words, emotion concepts, and emotional development in children: A constructionist hypothesis. Developmental Psychology, 55(9), 1830-1849. https://doi.org/10.1037/dev0000686.

Hoffman, P. (2018). An individual differences approach to semantic cognition: Divergent effects of age on representation, retrieval and selection. Scientific Reports, 8(8145), 110. https://doi.org/10.1038/s41598-018-26569-0.

Humphreys, G. F., Hoffman, P., Visser, M., Binney, R. J., \& Lambon Ralph, M. A. (2015). Establishing task- and modality-dependent dissociations between the semantic and default mode networks. PNAS, 112(25), 7857-7862. https://doi.org/10.1073/pnas.1422760112.

Itkes, O., Eviatar, Z., \& Kron, A. (2019). Semantic and affective manifestations of ambi (valence). Cognition and Emotion, 33(7), 1356-1369. https://doi.org/10.1080/02699931.2018.1564249. 
Itkes, O., \& Kron, A. (2019). Affective and semantic representations of valence: A conceptual framework. Emotion Review, 11(4), 283-293. https://doi.org/10.1177/1754073919868759.

Jackson, R. L. (2021). The neural correlates of semantic control revisited. NeuroImage, 224, 117444. https://doi.org/10.1016/j.neuroimage.2020.117444.

Jastorff, J., De Winter, F., Van den Stock, J., Vandenberghe, R., Giese, M. A., \& Vandenbulcke, M. (2016). Functional dissociation between anterior temporal lobe and inferior frontal gyrus in the processing of dynamic body expressions: Insights from behavioral variant frontotemporal dementia. Human Brain Mapping, 37, 4472-4486. https://doi.org/10.1002/hbm.23322.

Jefferies, E. (2013). The neural basis of semantic cognition: converging evidence from neuropsychology, neuroimaging and TMS. Cortex, 49, 611-625. https://doi.org/10.1016/j.cortex.2012.10.008.

Jefferies, E., \& Lambon Ralph, M. A. (2006). Semantic impairment in stroke aphasia versus semantic dementia: a case-series comparison. Brain, 129, 2132-2147. https://doi.org/10.1093/brain/aw1153.

Jefferies, E., Patterson, K., \& Lambon Ralph, M. A. (2008). Deficits of knowledge versus executive control in semantic cognition: Insights from cued naming. Neuropsychologia, 46, 649-658. https://doi.org/10.1016/j.neuropsychologia.2007.09.007.

Jefferies, E., Thompson, H., Cornelissen, P., \& Smallwood, J. (2019). The neurocognitive basis of knowledge about object identity and events: dissociations reflect opposing effects of semantic coherence and control. Philosophical Transactions of the Royal Society B, 375, 20190300. https://doi.org/10.1098/rstb.2019.0300. 
Kay, J., Lesser, R., \& Coltheart, M. (1992). Psycholinguistic assessments of language processing in aphasia (PALPA). Hove (UK): Lawrence Erlbaum Associates.

Kerschensteiner, M., Poeck, K., \& Brunner, E. (1972). The fluency-non fluency dimension in the classification of aphasic speech. Cortex, 8(2), 233-247. https://doi.org/10.1016/S0010-9452(72)80021-2.

Krieger-Redwood, K., Teige, C., Davey, J., Hymers, M., \& Jefferies, E. (2015). Conceptual control across modalities: graded specialisation for pictures and words in inferior frontal and posterior temporal cortex. Neuropsychologia, 76, 92-107. https://doi.org/10.1016/j.neuropsychologia.2015.02.030.

Kumfor, F., Ibañez, A., Hutchings, R., Hazelton, J. L., Hodges, J. R., \& Piguet, O. (2018). Beyond the face: how context modulates emotion processing in frontotemporal dementia subtypes. Brain, 141, 1172-1185. https://doi.org/10.1093/brain/awy002.

Kuznetsova, A., Brockhoff, P. B., \& Christensen, R. H. B. (2017). lmerTest package: Tests in linear mixed effects models. Journal of Statistical Software, 82(13), 1-26. https://doi.org/10.18637/jss.v082.i13.

Lambon Ralph, M. A., Jefferies, E., Patterson, K., \& Rogers, T. T. (2017). The neural and computational bases of semantic cognition. Nature Reviews Neuroscience, 18(1), 4255. https://doi.org/10.1038/nrn.2016.150.

Lanzoni, L., Ravasio, D., Thompson, H., Vatansever, D., Margulies, D., Smallwood, J., \& Jefferies, E. (2020). The role of default mode network in semantic cue integration. NeuroImage, 219, 117019. https://doi.org/10.1016/j.neuroimage.2020.117019.

Lanzoni, L., Thompson, H., Beintari, D., Berwick, K., Demnitz-King, H., Raspin, H., Taha, M., Stampacchia, S., Smallwood, J., \& Jefferies, E. (2019). Emotion and location cues 
bias conceptual retrieval in people with deficient semantic control. Neuropsychologia, 131, 294-305. https://doi.org/10.1016/j.neuropsychologia.2019.05.030.

Larsen, K., Petersen, J. H., Budtz-Jørgensen, E., \& Endahl, L. (2000). Interpreting parameters in the logistic regression model with random effects. Biometrics, 56(3), 909-914. https://doi.org/10.1111/j.0006-341x.2000.00909.x.

Lenth, R. (2020). Emmeans: Estimated Marginal Means, aka Least-Squares Means. R package version 1.5.2-1. https://CRAN.R-project.org/package=emmeans.

Leung, J. H., Purdy, S. C., Tippett, L. J., \& Leão, S. H. S. (2017). Affective speech prosody perception and production in stroke patients with left-hemispheric damage and healthy controls. Brain \& Language, 166, 19-28. https://doi.org/10.1016/j.bandl.2016.12.001.

Lindquist, K. A., Barrett, L. F., Bliss-Moreau, E., \& Russell, J. A. (2006). Language and the perception of emotion. Emotion, 6(1), 125-138. https://doi.org/10.1037/15283542.6.1.125.

Lindquist, K. A., Gendron, M., Barrett, L. F., \& Dickerson, B. C. (2014). Emotion perception, but not affect perception, is impaired with semantic memory loss. Emotion, 14(2), 375-387. https://doi.org/10.1037/a0035293.

Lindquist, K. A., Gendron, M., \& Satpute, A. B. (2016). Language and emotion: Putting words into feelings and feelings into words. In L. F. Barrett, M. Lewis, \& J. M. Haviland-Jones (Eds.). Handbook of emotions $\left(4^{\text {th }}\right.$ ed., pp. 579-594). New York, NY: Guildford Press. 
Lindquist, K. A., Satpute, A. B., \& Gendron, M. (2015). Does language do more than communicate emotion? Current Directions in Psychological Science, 24(2), 99-108. https://doi.org/10.1177/0963721414553440.

Marcus, D. S., Fotenos, A. F., Csernansky, J. G., Morris, J. C., \& Buckner, R. L. (2010). Open access series of imaging studies: Longitudinal MRI data in nondemented and demented older adults. Journal of Cognitive Neuroscience, 22(12), 2677-2684. https://doi.org/10.1162/jocn.2009.21407.

Miller, L. A., Hsieh, S., Lah, S., Savage, S., Hodges, J. R., \& Piguet, O. (2012). One size does not fit all: Face emotion processing impairments in semantic dementia, behavioural-variant frontotemporal dementia and Alzheimer's disease are mediated by distinct cognitive deficits. Behavioural Neurology, 25, 53-60. https://doi.org/10.1155/2012/683052.

Montefinese, M., Hallam, G., Stampacchia, S., Thompson, H. E., \& Jefferies, E. (2020). Deficits of semantic control disproportionately affect low-relevance conceptual features: evidence from semantic aphasia. Aphasiology. https://doi.org/10.1080/02687038.2020.1814950.

Mummery, C. J., Patterson, K., Price, C. J., Ashburner, J., Frackowiak, R. S., \& Hodges, J. R. (2000). A voxel-based morphometry study of semantic dementia: relationship between temporal lobe atrophy and semantic memory. Annals of Neurology, 47(1), 36-45. https://doi.org/10.1002/1531-8249(200001)47:1<36::AID-ANA8>3.0.CO;2-L.

Nook, E. C., Lindquist, K. A., \& Zaki, J. (2015). A new look at emotion perception: concepts speed and shape facial emotion recognition. Emotion, 15(5), 569-578. https://doi.org/10.1037/a0039166. 
Noonan, K. A, Jefferies, E., Corbett, F., \& Lambon Ralph, M. A. (2010). Elucidating the nature of deregulated semantic cognition in semantic aphasia: Evidence for the roles of prefrontal and temporo-parietal cortices. Journal of Cognitive Neuroscience, 22(7), 1597-1613. https://doi.org/10.1162/jocn.2009.21289.

Noonan, K. A., Jefferies, E., Visser, M., \& Lambon Ralph, M. A. (2013). Going beyond inferior prefrontal involvement in semantic control: evidence for the additional contribution of dorsal angular gyrus and posterior middle temporal cortex. Journal of Cognitive Neuroscience, 25(11), 1824-1850. https://doi.org/10.1162/jocn_a_00442.

Osborne-Crowley, K., Wilson, E., De Blasio, F., Wearne, T., Rushby, J., \& McDonald, S. (2019). Preserved rapid conceptual processing of emotional expressions despite reduced neuropsychological performance following traumatic brain injury. Neuropsychology, 33(6), 872-882. https://doi.org/10.1037/neu0000545.

Osborne-Crowley, K., Wilson, E., De Blasio, F., Wearne, T., Rushby, J., \& McDonald, S. (2020). Empathy for people with similar experiences: Can the perception-action model explain empathy impairments after traumatic brain injury? Journal of Clinical and Experimental Neuropsychology, 42(1), 28-41.

\section{https://doi.org/10.1080/13803395.2019.1662375.}

Patterson, K., Nestor, P. J., \& Rogers, T. T. (2007). Where do you know what you know? The representation of semantic knowledge in the human brain. Nature Reviews Neuroscience, 8, 976-987. https://doi.org/10.1038/nrn2277.

Peirce, J. W., Gray, J. R., Simpson, S., MacAskill, M. R., Höchenberger, R., Sogo, H., Kastman, E., \& Lindeløv, J. (2019). PsychoPy2: experiments in behavior made easy. Behavior Research Methods, 51, 195-203. https://doi.org/10.3758/s13428-018-01193y. 
Phan, T. G., Fong, A. C., Donnan, G. A., \& Reutens, D. C. (2007). Digital map of posterior cerebral artery infarcts associated with posterior cerebral artery trunk and branch occlusion. Stroke, 38, 1805-1811. https://doi.org/10.1161/STROKEAHA.106.477000.

Preston, S. D. (2007). A perception-action model for empathy. In T. F. D. Farrow (Ed.), Empathy in mental illness (pp. 428-447). Cambridge: Cambridge University Press.

R Core Team (2020). R: A language and environment for statistical computing. R Foundation for Statistical Computing, Vienna, Austria. URL: https://www.R-project.org/.

Raven, J. (1962). Coloured progressive matrices sets A, AB, B. London: H.K. Lewis.

Reitan, R. M. (1958). Validity of the trail making test as an indicator of organic brain damage. Perceptual and Motor Skills, 8, 271-276.

\section{https://doi.org/10.2466/pms.1958.8.3.271.}

Riberto, M., Pobric, G., \& Talmi, D. (2019). The emotional facet of subjective and neural indices of similarity. Brain Topography, 32, 956-964. https://doi.org/10.1007/s10548019-00743-7.

Robertson, D., Davidoff, J., \& Braisby, N. (1999). Similarity and categorisation: neuropsychological evidence for a dissociation in explicit categorisation tasks. Cognition, 71, 1-42. http://dx.doi.org/10.1016/S0010-0277(99)00013-X.

Robertson, I., Ward, T., Ridgeway, V., \& Nimmo-Smith, I. (1994). The test of everyday attention. London: Thames Valley Test Company.

Rogers, T. T., Patterson, K., Jefferies, E., \& Lambon Ralph, M. A. (2015). Disorders of representation and control in semantic cognition: Effects of familiarity, typicality, and specificity. Neuropsychologia, 76, 220-239.

https://doi.org/10.1016/j.neuropsychologia.2015.04.015. 
Rosen, H. J., Gorno-Tempini, M. L., Goldman, W. P., Perry, R. J., Schuff, N., Weiner, M., Feiwell, R., Kramer, J. H., \& Miller, B. L. (2002). Patterns of brain atrophy in frontotemporal dementia and semantic dementia. Neurology, 58(2), 198-208. https://doi.org/10.1212/WNL.58.2.198.

Ross, E. D., Thompson, R. D., \& Yenkosky, J. (1997). Lateralization of affective prosody in brain and the callosal integration of hemispheric language functions. Brain and Language, 56, 27-54. https://doi.org/10.1006/brln.1997.1731.

Samson, D., Connolly, C., \& Humphreys, G. W. (2007). When "happy" means "sad": Neuropsychological evidence for the right prefrontal cortex contribution to executive semantic processing. Neuropsychologia, 45, 896-904. https://doi.org/10.1016/j.neuropsychologia.2006.08.023.

Sauter, D. A. (2018). Is there a role for language in emotion perception? Emotion Review, 10(2), 111-115. https://doi.org/10.1177/1754073917693924.

Souter, N. E., Stampacchia, S., Hallam, G., Thompson, H., Smallwood, J., \& Jefferies, E. (Unpublished results). Motivated semantic control: Exploring the effects of extrinsic reward and self-reference on semantic retrieval in semantic aphasia. BioRxiv. https://doi.org/10.1101/2021.05.25.444996.

Stampacchia, S., Thompson, H. E., Ball, E., Nathaniel, U., Hallam, G., Smallwood, J., Lambon Ralph, M. A., \& Jefferies, E. (2018). Shared processes resolve competition within and between episodic and semantic memory: evidence from patients with LIFG lesions. Cortex, 108, 127-143. https://doi.org/10.1016/j.cortex.2018.07.007.

Thompson, H. E., Almaghyuli, A., Noonan, K. A., barak, O., Lambon Ralph, M. A., \& Jefferies, E. (2018). The contribution of executive control to semantic cognition: 
Convergent evidence from semantic aphasia and executive dysfunction. Journal of Neuropsychology, 12, 312-340. https://doi.org/10.1111/jnp.12142.

Thompson, H., Davey, J., Hoffman, P., Hallam, G., Kosinski, R., Howkins, S., Wooffindin, E., Gabbitas, R., \& Jefferies, E. (2017). Semantic control deficits impair understanding of thematic relationships more than object identity. Neuropsychologia, 104, 113-125. https://doi.org/10.1016/j.neuropsychologia.2017.08.013.

Thompson, H. E., Henshall, L., \& Jefferies, E. (2016). The role of the right hemisphere in semantic control: A case-series comparison of right and left hemisphere stroke. Neuropsychologia, 85, 44-61. https://doi.org/10.1016/i.neuropsychologia.2016.02.030.

Thompson-Schill, S. L., D’Esposito, M., Aguirre, G. K., \& Farah, M. J. (1997). Role of left inferior prefrontal cortex in retrieval of semantic knowledge: A reevaluation. PNAS, 94(26), 14792-14797. https://doi.org/10.1073/pnas.94.26.14792.

Tracy, J. L., \& Robins, R. W. (2008). The automaticity of emotion recognition. Emotion, 8(1), 81-95. https://doi.org/10.1037/1528-3542.8.1.81.

Vatansever, D., Bzdok, D., Wang, H-T., Mollo, G., Sormaz, M., Murphy, C., Karapanagiotidis, T., Smallwood, J., \& Jefferies, E. (2017). Varieties of semantic cognition revealed through simultaneous decomposition of intrinsic brain connectivity and behaviour. NeuroImage, 158, 1-11. https://doi.org/10.1016/j.neuroimage.2017.06.067.

Wang, X., Margulies, D. S., Smallwood, J., \& Jefferies, E. (2020). A gradient from long-term memory to novel cognition: Transitions through default mode and executive cortex. NeuroImage, 220, 117074. https://doi.org/10.1016/j.neuroimage.2020.117074. 
Warrington, E. K., \& James, M. (1991). The Visual Object and Space Battery Perception. Bury St Edmunds: Thames Valley Company.

Wechsler, D. (1997). Wechsler memory scale (3rd ed.). San Antonio, TX: The Psychological Corporation.

Zemack-Rugar, Y., Bettman, J. R., \& Fitzsimons, G. J. (2007). The effects of nonconsciously priming emotion concepts on behavior. Journal of Personality and Social Psychology, 93(6), 927-939. https://doi.org/10.1037/0022-3514.93.6.927.

Zhang, M., Varga, D., Wang, X., Krieger-Redwood, K., Gouws, A., Smallwood, J., \& Jefferies, E. (2021). Knowing what you need to know in advance: The neural processes underpinning flexible semantic retrieval of thematic and taxonomic relations. NeuroImage, 224, 117405.

https://doi.org/10.1016/j.neuroimage.2020.117405.

Zhou, P., Critchley, H., Garfinkel, S., \& Gao, Y. (2021). The conceptualization of emotions across cultures: a model based on interoceptive neuroscience. Neuroscience and Biobehavioral Reviews, 125, 314-327. https://doi.org/10.1016/j.neubiorev.2021.02.023.

Zoom Video Communications Inc. (2016). Security guide. Zoom Video Communications Inc. Retrieved from https://d24cgw3uvb9a9h.cloudfront.net/static/81625/doc/ZoomSecurity-White-Paper.pdf. 